\title{
MdWRKY75e enhances resistance to Alternaria alternata in Malus domestica
}

\author{
Yingjun Hou (1)', Xinyi Yu', Weiping Chen², Weibing Zhuang ${ }^{3}$, Sanhong Wang ${ }^{1}$, Chao Sun', Lifang Cao', \\ Tingting Zhou ${ }^{1}$ and Shenchun Qu ${ }^{1 凶}$
}

\begin{abstract}
The Alternaria alternata apple pathotype adversely affects apple (Malus domestica Borkh.) cultivation. However, the molecular mechanisms underlying enhanced resistance to this pathogen in apple remain poorly understood. We have previously reported that MdWRKY75 expression is upregulated by A. alternata infection in 'Sushuai' apples. In this study, we discovered that overexpression of MdWRKY75e increased the resistance of transgenic apple lines to $A$. alternata infection, whereas silencing this gene enhanced susceptibility to A. alternata infection. Furthermore, we found that MdWRKY75e directly binds to the MdLAC7 promoter to regulate the biosynthesis of laccase and increase the biosynthesis of lignin during A. alternata infection. Moreover, the thickening of the cell wall enhanced the mechanical defense capabilities of apple. In addition, we found that jasmonic acid remarkably induced MdWRKY75e expression, and its levels in transgenic apple lines were elevated. These results indicate that MdWRKY75e confers resistance to the A. alternata apple pathotype mainly via the jasmonic acid pathway and that pathogenesis-related genes and antioxidant-related enzyme activity are involved in the disease resistance of MdWRKY75e transgenic plants. In conclusion, our findings provide insights into the importance of MdWRKY75e for resistance to A. alternata infection in apples.
\end{abstract}

\section{Introduction}

Apple (Malus domestica) is a globally cultivated fruit crop species. However, apple cultivation is adversely affected by various fungi, including those caused by the Alternaria alternata apple pathotype (Alternaria alternata f. sp. mali) ${ }^{1-3}$. Current $A$. alternata management practices include conventional chemical treatments with adverse side effects, such as environmental pollution and food safety hazards. The most effective strategy for fighting fungal diseases in apple is the selective breeding of resistant cultivars ${ }^{2,4}$. However, the mechanism of resistance to apple leaf spot disease in apples has not been extensively studied. Therefore, it is important to

\footnotetext{
Correspondence: Shenchun Qu (qscnj@njau.edu.cn)

${ }^{1}$ College of Horticulture, Nanjing Agricultural University, Nanjing, People's Republic of China

${ }^{2}$ College of Agriculture, Nanjing Agricultural University, Nanjing, People's Republic of China

Full list of author information is available at the end of the article
}

investigate the potential molecular mechanism underlying resistance to apple leaf spot disease.

Plants possess two major innate immune responses for defense against pathogens ${ }^{5}$. One response involves pattern recognition receptors (PRRs) that recognize pathogen-associated molecular patterns (PAMPs), and the stimulation of PRRs results in PAMP-triggered immunity to activate a basal defense response; the other involves effector-triggered immunity, which induces the expression of resistance gene products that specifically recognize the effectors delivered by pathogens to weaken PAMPtriggered immunity ${ }^{6-9}$. The two innate immune systems partially work together to trigger downstream responses, such as the generation of plant hormones, reactive oxygen species (ROS), and secondary metabolites, and the induction of a hypersensitive response to activate the corresponding defense systems ${ }^{10}$.

Various plant hormones, such as salicylic acid (SA), ethylene (ET), and jasmonic acid (JA), enhance plant

\section{(c) The Author(s) 2021}

(c) Open Access This article is licensed under a Creative Commons Attribution 4.0 International License, which permits use, sharing, adaptation, distribution and reproduction cc) in any medium or format, as long as you give appropriate credit to the original author(s) and the source, provide a link to the Creative Commons license, and indicate if changes were made. The images or other third party material in this article are included in the article's Creative Commons license, unless indicated otherwise in a credit line to the material. If material is not included in the article's Creative Commons license and your intended use is not permitted by statutory regulation or exceeds the permitted use, you will need to obtain permission directly from the copyright holder. To view a copy of this license, visit http://creativecommons.org/licenses/by/4.0/. 
defense responses against pathogens and abiotic stress $^{11,12}$. SA is principally involved in systemic acquired resistance as a mediator of plant defense responses to biotrophic and hemibiotrophic pathogens, while JA and ET interact with each other to activate defense responses against necrotrophic pathogens. The interactions between JA and SA signaling pathways are antagonistic rather than cooperative ${ }^{13}$. Both the SA and JA pathways are stimulated by pathogen infection, leading to the accumulation of endogenous SA and JA, which act as signaling molecules for transcriptional responses of pathogen-associated genes $^{14,15}$. JASMONATE INSENSITIVE 1/MYC2 (JIN1/ MYC2), a transcription factor, mediates JA-responsive gene expression to trigger defense responses in host plants ${ }^{16}$. The JA-responsive marker genes thionin (Thi2.1) and defensin (PDF1.2) accumulate in plants challenged by fungal pathogens ${ }^{17,18}$. Under biotic and abiotic stresses, plants can improve the activities of antioxidant enzymes, such as catalase (CAT), superoxide dismutase (SOD), and peroxidase (POD), which scavenge ROS, particularly $\mathrm{H}_{2} \mathrm{O}_{2}$ and $\mathrm{O}_{2}{ }^{-}$, to reduce their accumulation and mitigate their damage by maintaining dynamic equilibrium ${ }^{19,20}$. The enzyme phenylalanine ammonia-lyase (PAL) is critical for SA synthesis pathways that limit biotic and abiotic stresses ${ }^{21}$. Additionally, PAL has an important role in lignin synthesis via the phenylpropanoid pathway, which is the most common secondary metabolic pathway involved in plant defense reactions against abiotic and biotic stresses; the polymerization of monolignol to form lignin provides mechanical strength and reinforces plant cell walls, providing a physical barrier against pathogens and pests ${ }^{22,23}$. The polymerization of monolignols into lignin polymers is catalyzed either by peroxidases or laccases $^{24}$. In apple, the molecular mechanisms underlying the genetic responses conferring resistance to fungal infections are still unclear.

The WRKY transcription factor family in plants plays an important role in signaling pathways that respond to biotic (fungi, pathogenic bacteria, oomycetes, and viruses) and abiotic (heat, soil salinity, oxidative stress, drought, cold, irradiation, and heavy metals) stresses. The WRKY protein structural domain comprises a 60 -amino acid-long DNA bonding domain characterized by a highly conserved $\mathrm{N}$-terminal core known as the WRKYGQK motif. WRKYs are divided into three major groups (I, II, and III), where group II is further categorized into five subgroups (IIa, IIb, IIc, IId, and IIe) according to the number of WRKY structural domains and the structure of the zinc finger motif ${ }^{25,26}$. Studies on Arabidopsis have revealed that AtWRKY75 overexpression enhances resistance to Pectobacterium carotovora ssp. Carotovora (Pcc) via SA and JA defense signaling ${ }^{27}$. Similarly, AtWRKY75 decreases the severity of Pcc-induced bacterial soft rot and activates a subset of defense-related genes against Pcc infection in Chinese cabbage $^{28}$. AtWRKY75 and AtWRKY28 overexpression strengthens the resistance to oxalic acid and Sclerotinia sclerotiorum stress, mainly via the JA/Eth pathway ${ }^{29}$. AtWRKY75 interacts with DELLA proteins, which affect flowering via the GA-mediated signaling pathway in Arabidopsis $^{30}$ and induce leaf senescence by interacting with SA and $\operatorname{ROS}^{31}$. PtrWRKY75 overexpression enhances drought tolerance through salicylic acid-induced ROS accumulation in poplar ${ }^{32}$. Additionally, PagWRKY75 can negatively regulate salt and osmotic tolerance by modulating various physiological processes in poplar ${ }^{33}$. In apple, MdWRKY100 overexpression increases resistance to Colletotrichum gloeosporioides, while its silencing by RNAi rendered the transgenic plants more sensitive to this pathogen ${ }^{34}$. MdWRKY33 contributes to resistance against Pythium ultimum infection in apple roots ${ }^{35}$. MdWRKY15, MdWRKY31, and MdWRKY46 enhance resistance to Botryosphaeria dothidea in apple by activating the expression of MdICS1, MdHIR4, and $M d P B S 3.1$, respectively, in the SA biosynthesis pathway $^{36-38}$.

Herein, we found that MdWRKY75e was significantly induced by $A$. alternata and MeJA treatments. However, MdWRKY75e expression was significantly inhibited by SA treatment, and MdWRKY75e was directly bound to the MdLAC7 gene promoter. We further show that MdWRKY75e is a positive regulator of MdLAC7 expression as well as laccase and lignin biosynthesis. Moreover, our findings suggest that MdWRKY75e acts as a positive modulator of disease resistance, at least in part, by regulating antioxidant enzyme-mediated ROS scavenging. Our study reveals the molecular mechanism underlying the role of WRKYs in apple resistance to fungal diseases and provides a candidate gene for breeding diseaseresistant apples.

\section{Results \\ Isolation and bioinformatics analysis of MdWRKY75s}

We previously identified the infection-induced WRKY TFs MdWRKY 75d and MdWRKY75e in the transcriptome of 'Sushuai' apple. MdWRKY75d sequencing revealed that the full length of the cDNA was $666 \mathrm{bp}$. Bioinformatics analysis indicated that MdWRKY75d encodes a putative peptide consisting of 221 amino acids, with an isoelectric point of 9.12 and a molecular mass of $25.5 \mathrm{kDa}$. MdWRKY75e sequencing showed that the full length of its cDNA was $453 \mathrm{bp}$, and bioinformatics analysis suggested that it encoded a putative peptide composed of 150 amino acids, with an isoelectric point of 9.56 and a molecular mass of $17.1 \mathrm{kDa}$. Multiple sequence alignment suggested that MdWRKY75d and MdWRKY75e had a highly conserved sequence, including a WRKYGQK structural domain followed by a zinc finger-like motif (Fig. 1a). The 
(a)

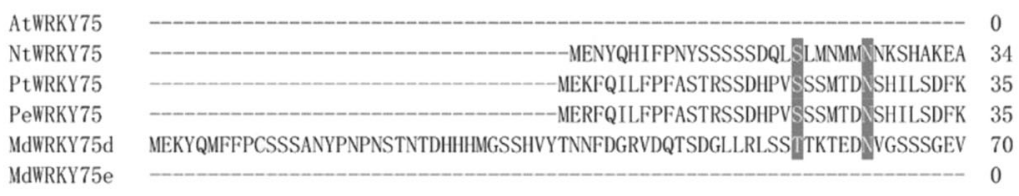

AtWRKY75

NtWRKY75

PtWRKY75

PeWRKY75

MdWRKY75d

MdWRKY75e

AtWRKY75

NtWRKY75

PtWRKY75

PeWRKY75

MdWRKY75d

MdWRKY75e

AtWRKY75

NtWRKY75

PtWRKY75

PeWRKY75

MdWRKY75d

MdWRKY75e
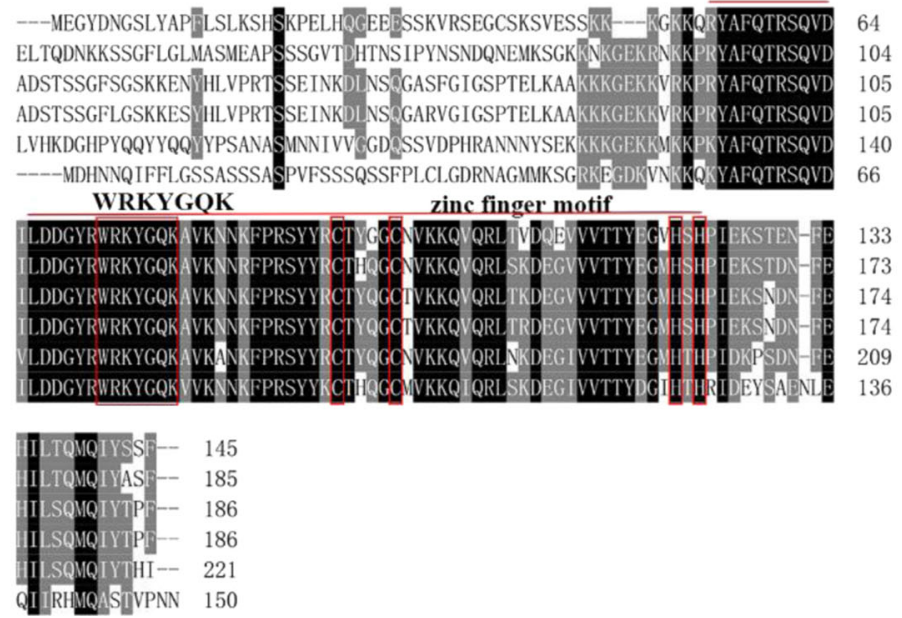

(b)

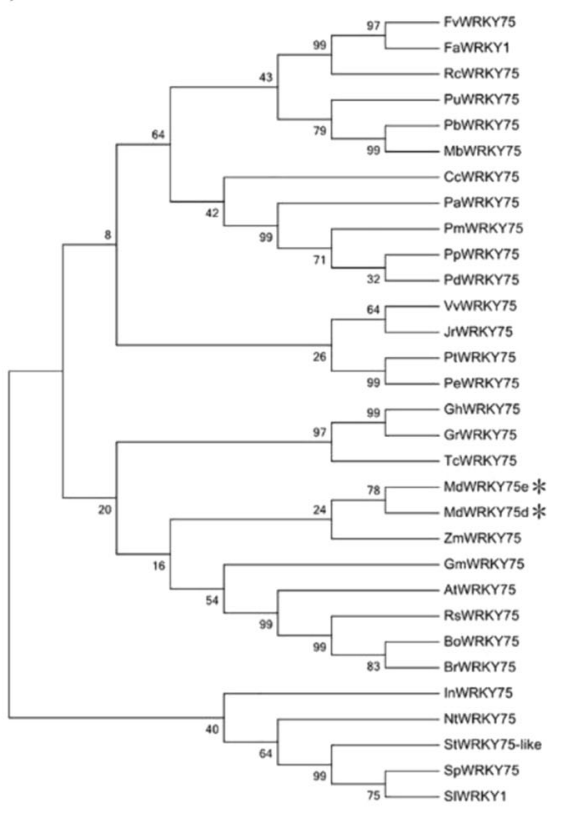

Fig. 1 Multiple alignments of MdWRKY75d and MdWRKY75e and phylogenetic analysis of WRKY75s from different plants. a Amino acid sequence alignment of MdWRKY75d, MdWRKY75e, and WRKY75s from various plant species. The same amino acids are displayed on a black background, and similar amino acids are shown in gray. The WRKY motif is indicated by a line, and the zinc finger structure is marked by a red circle. The sequences used for comparison with MdWRKY75d and MdWRKY75e were Arabidopsis thaliana AtWRKY75 (NP_196812.1), Nicotiana tabacum NtWRKY75 (XP_016446514.1), Populus trichocarpa PtWRKY75 (XP_006373216.1), and Populus euphratica PeWRKY75 (XP_01 1009191.1). b Construction of a phylogenetic tree on the basis of WRKYs from different plants, including MdWRKY75d and MdWRKY75e. A phylogenetic tree was created with MdWRKY75d, MdWRKY75e, and the following WRKY75 family proteins: Vitis vinifera VwWRKY75 (XP_002275576.1), Populus trichocarpa PtWRKY75 (XP_006373216.1), Populus euphratica PeWRKY75 (XP_011009191.1), Nicotiana tabacum NtWRKY75 (XP_016446514.1), Arabidopsis thaliana AtWRKY75 (NP_196812.1), Raphanus sativus RsWRKY75 (XP_018475395.1), Brassica oleracea var. oleracea BoWRKY75 (XP_013626795.1), Brassica rapa BrWRKY75 (XP_009131315.1), Rosa chinensis RcWRKY75 (XP_024197588.1), Fragaria vesca subsp. vesca FvWRKY75 (XP_004310100.1), Pyrus X bretschneideri PbWRKY75 (XP_009372253.1), Fragaria $\times$ ananassa FaWRKY1 (ACH88751.1), Ipomoea nil InWRKY75 (XP_019199847.1), Theobroma cacao TcWRKY75 (XP_007031915.1), Malus baccata MbWRKY75 (AHJ78583.1), Juglans regia JrWRKY75 (XP_018836802.1), Prunus avium PaWRKY75 (XP_021828982.1), Gossypium hirsutum GhWRKY75 (XP_016725404.1), Pyrus ussuriensis $\times$ Pyrus communis PuWRKY75 (KAB2599104.1), Gossypium raimondii GrWRKY75 (XP_012446088.1), Prunus mume PmWRKY75 (XP_008222197.1), Prunus persica PpWRKY75 (XP_007226197.1), Prunus dulcis PdWRKY75

(XP_034198908.1), Citrus clementina CcWRKY75 (XP_006437986.1), Solanum tuberosum StWRKY75-like (NP_001275604.1), Solanum pennellii SpWRKY75 (XP_015075730.1), Solanum lycopersicum SIWRKY1 (NP_001310244.1), Glycine max GmWRKY75 (XP_003549123.1), and Zea mays ZmWRKY75 (PWZ27788.1). Bootstrap values are shown for each diverging branch. MdWRKY75d and MdWRKY75d are denoted with asterisks

phylogenetic tree constructed with MdWRKY75d and MdWRKY75e, as well as a total of 29 WRKYs from different plants, showed that these TFs are most closely related to ZmWRKY75 (Fig. 1b).

\section{Expression of MdWRKY75s under different treatments and in various organisms}

The expression patterns of MdWRKY75d and MdWRKY75e under infection with pathogens, MeJA and SA treatments were examined by RT-qPCR (unpublished article). As shown in Supplementary Fig. S1a-c, $M d W R K Y 75 d$ and MdWRKY75e transcript levels were significantly induced by $A$. alternata and MeJA treatment. However, MdWRKY75d and MdWRKY75e expression was significantly inhibited by SA treatment.
MdWRKY75d and MdWRKY75e transcripts were detected in all organs of the apple plants. The abundance of MdWRKY75d transcripts was high in the flowers and ripe fruits, moderate in the stems and young fruits, and low in young and old leaves. Conversely, the abundance of MdWRKY75e transcripts was high in young leaves, moderate in old leaves, ripe fruits, and flowers, and low in stems and young fruits (Supplementary Fig. S1d).

\section{Subcellular localization of MdWRKY75s}

Fusion plasmids for 35S::GFP-MdWRKY75d and 35S:: GFP-MdWRKY75e were generated by fusing the full length of MdWRKY75d and MdWRKY75e to the N terminus of the GFP reporter protein using the pCAMBIA1302 vector driven by the CaMV 35S promoter. Next, 
35S::GFP-MdWRKY75d, 35S::GFP-MdWRKY75e, and a nuclear marker (35S::D53-RFP construct; positive control) were cotransformed into Nicotiana benthamiana leaves by Agrobacterium-mediated transient transformation ${ }^{39}$. Microscopic observation showed that the 35S::GFP vector was present in the cytoplasm and nucleus of tobacco cells, while the MdWRKY75d-GFP and MdWRKY75e-GFP fusion proteins were only present in the nucleus (Fig. 2a). Together, these data suggest that MdWRKY75d and MdWRKY75e localize to the nucleus.

\section{Transactivation activity of MdWRKY75s}

The fusion plasmids were generated by fusing the fulllength coding regions of MdWRKY75d and MdWRKY75e with the DNA binding domain of GAL4 using the pGBKT7 vector and transformed into the yeast strain AH109. The growth of the fusion plasmid-containing yeast cells was comparable to that of yeast containing the control plasmid (pGBKT7) in the same selection medium (SD)/Leu/Trp. Yeast cells transformed with any vector grew on SD/-Leu/-Trp medium, showing that this system was reliable. Yeast cells transformed with the negative control plasmid pGBKT7 failed to grow on SD/-Leu/Trp/-His medium, while cells transformed with the reconstituted vector survived on a selection medium supplemented with $50 \mathrm{mM}$ 3-amino-1,2,4-triazole (3-AT). Furthermore, only yeast cells transformed with the fusion plasmid changed to blue when cultivated on SD/Leu/Trp/ His medium supplemented with both $50 \mathrm{mM} 3-\mathrm{AT}$ and $20 \mathrm{mM} \mathrm{X}$-a-Gal (Fig. 2b). These results indicated that MdWRKY75d and MdWRKY75e have transactivation activity in yeast.

\section{Overexpression of MdWRKY75e modulated laccase biosynthesis and altered the endogenous lignin contents in transgenic plants}

Under the control of the CaMV $35 \mathrm{~S}$ promoter, $M d W R K Y 75 d-$ and MdWRKY75e-overexpressing transgenic tobacco was generated by Agrobacterium-mediated transformation (Supplementary Fig. S2A). In total, eight transformants ( $\mathrm{T}_{0}$ generation) of MdWRKY75d and MdWRKY75e were confirmed as positive lines by GUS staining. Semiquantitative RT-PCR analysis showed that MdWRKY75d and MdWRKY75e were overexpressed in six and seven lines, respectively. Based on the real-time qPCR results (Supplementary Table S1), three independent overexpression lines of tobacco from among the six $M d W R K Y 75 d$-overexpressing lines, designated \#18, \#25, and \#36, and another three from the seven MdWRKY75eoverexpressing lines, named \#9, \#32, and \#36, were selected for the following assays, together with the corresponding untransformed wild-type (WT) and empty vector-transformed WT (empty vector) lines (Supplementary Fig. S2B, C).
Forty-five-days-old WT, empty vector, and transgenic plants with consistent growth were infected with the pathogen for $48 \mathrm{~h}$. Interestingly, the WT and empty vector plants showed greater sensitivity to infection with pathogens than $M d W R K Y 75 e$ transgenic plants. In particular, lesion sizes in $M d W R K Y 75$ e transgenic lines were particularly small after $24 \mathrm{~h}$ of infection with pathogens, and this result was further confirmed by the disease index (Fig. 3a, b). In contrast, lesion sizes in MdWRKY75d transgenic plants were not significantly different compared to those in the WT and empty vector plants (Supplementary Fig. S3a, b). Therefore, we further found that the growth phenotypes and root lengths of MdWRKY75e transgenic plants were not markedly different from those of the WT and empty vector plants (Fig. 3c). Compared to the WT and empty vector plants, the roots of transgenic MdWRKY75e plants had a deeper color and increased lignification (Fig. 3d). Furthermore, the dry weights of leaves and roots were heavier in transgenic MdWRKY75e plants than in the WT and empty vector plants (Fig. 3e, f). As shown in Fig. 3g-j, NtLAC7 expression and laccase activity in MdWRKY75e transgenic plants were significantly higher than those in WT and empty vector plants. Moreover, the lignin contents in the leaves and roots of MdWRKY75e transgenic lines were markedly higher than those of WT and empty vector plants.

Three transgenic GL-3 lines (designated \#1, \#8, and \#12) (Supplementary Fig. S4A) were examined by GUS staining, semi-quantitative RT-PCR, and qRT-PCR analyses (Supplementary Fig. S4B). Exposure of 45-days-old WT, empty vector, and transgenic GL-3 plants (Supplementary Fig. S4C) to disease stress caused by infection with pathogens for $48 \mathrm{~h}$ showed that the WT and empty vector plants had significantly larger lesion areas in leaves than the transgenic plants, further confirming the disease index results (Fig. 4a, b). Paraffin sectioning with toluidine blue $\mathrm{O}$ staining to compare the physiological differences among transgenic, WT, and empty vector plants showed that the upper epidermal cell wall was thickened in the transgenic line but not in the others. Moreover, wound and mycelium formation in transgenic lines was significantly smaller than that in the WT and empty vector plants after $24 \mathrm{~h}$ of infection with pathogens (Fig. 4c). Moreover, the expression levels of Alta1 and GAPdh in A. alternata were considerably lower in the transgenic plants than in the WT and empty vector plants (Fig. 4d, e).

Interestingly, root callus formation and lignification in transgenic plants were noticeably higher than those in WT and empty vector plants, consistent with the growth phenotype. Furthermore, MdWRKY75e overexpression suppressed root hair development, lateral root length and number, and root hair number (Fig. 5a). We further found that root dry weight was higher in transgenic plants than 


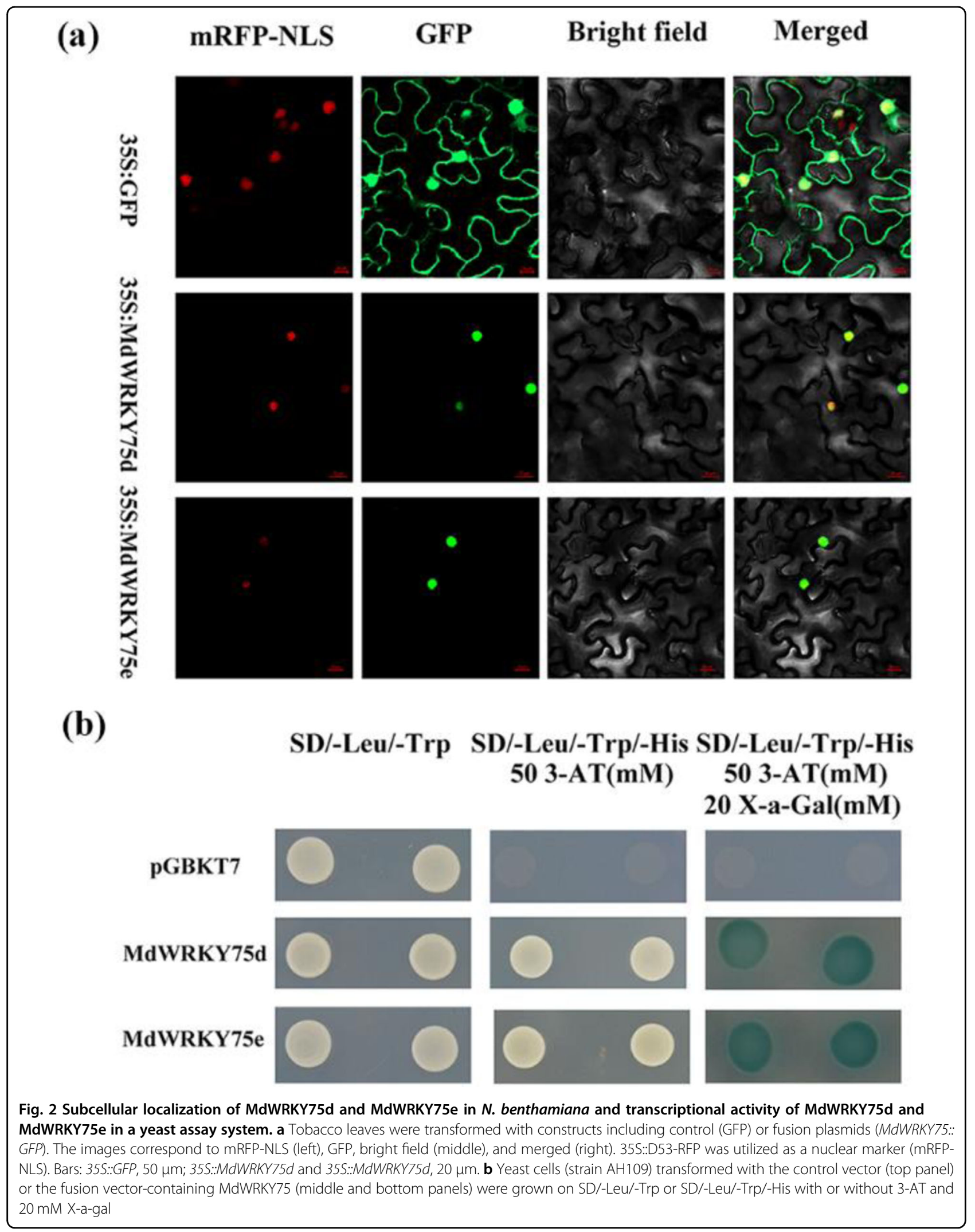




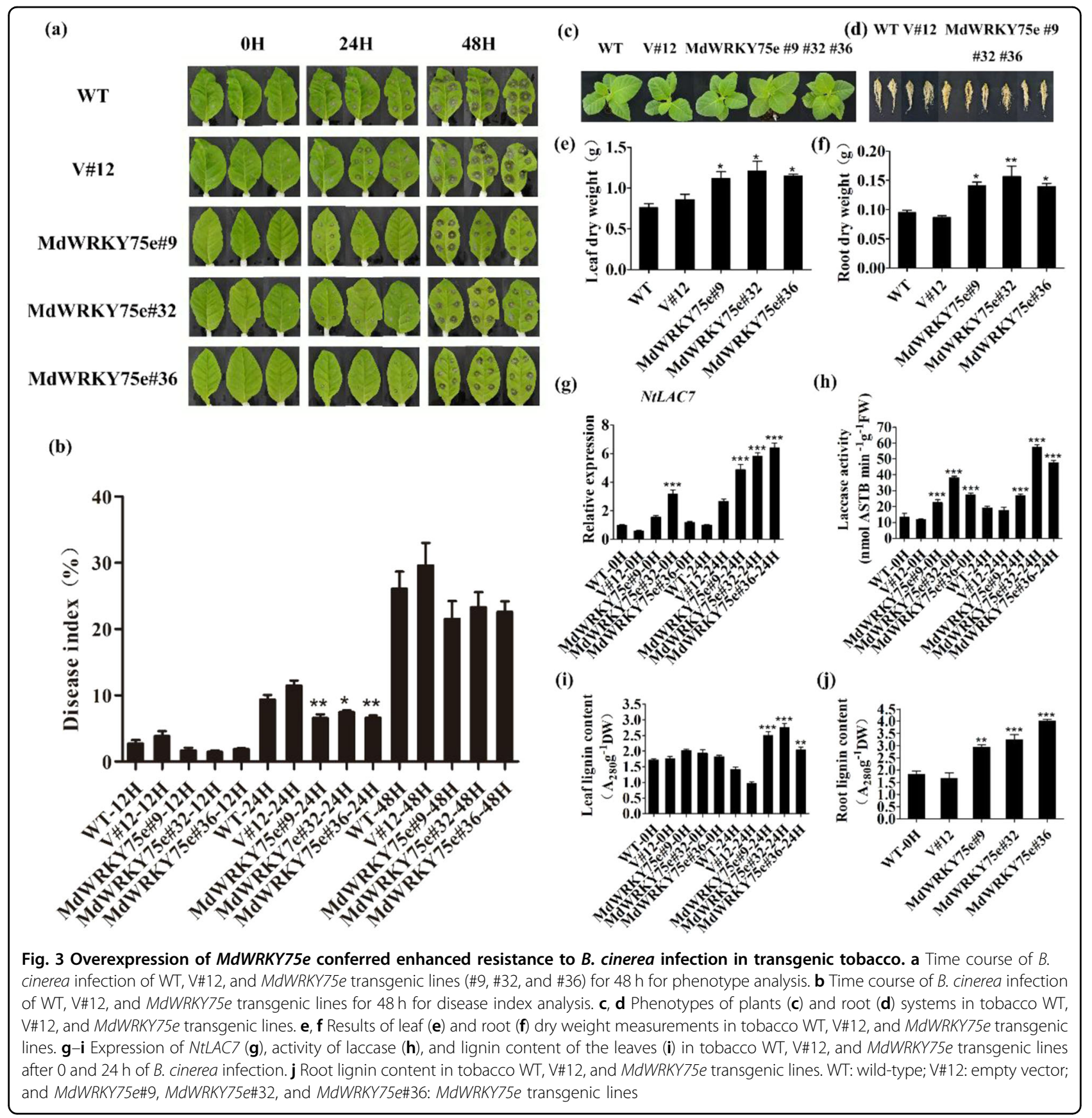

in WT and empty vector plants; in contrast, leaf dry weight was slightly higher in transgenic plants than in WT and empty vector plants (Fig. 5b, c). MdLAC7, MdPR9, $M d R F K 1$, and $M d W R K 1$ expression was strongly induced in MdWRKY75e-overexpressing lines after pathogen infection (Fig. $5 \mathrm{~d}-\mathrm{g}$ ). Furthermore, we attempted to determine whether laccase activities were altered in the tested plants and found them to be progressively higher in the transgenic lines than in the WT and empty vector lines (Fig. 5h). Additionally, we found that the lignin contents of leaves and roots in GL-3 transgenic lines were remarkably higher than those in WT and empty vector plants (Fig. 5i, j). In summary, these data showed that MdWRKY75e overexpression improved endogenous laccase activities and lignin contents and enhanced disease tolerance in transgenic plants.

\section{MdWRKY75e silencing in GL-3 confers sensitivity to pathogen infection}

We attempted to knock down MdWRKY75e in GL-3 transgenic lines by virus-induced gene silencing (VIGS). We constructed a pTRV2-GFP vector and reliably 


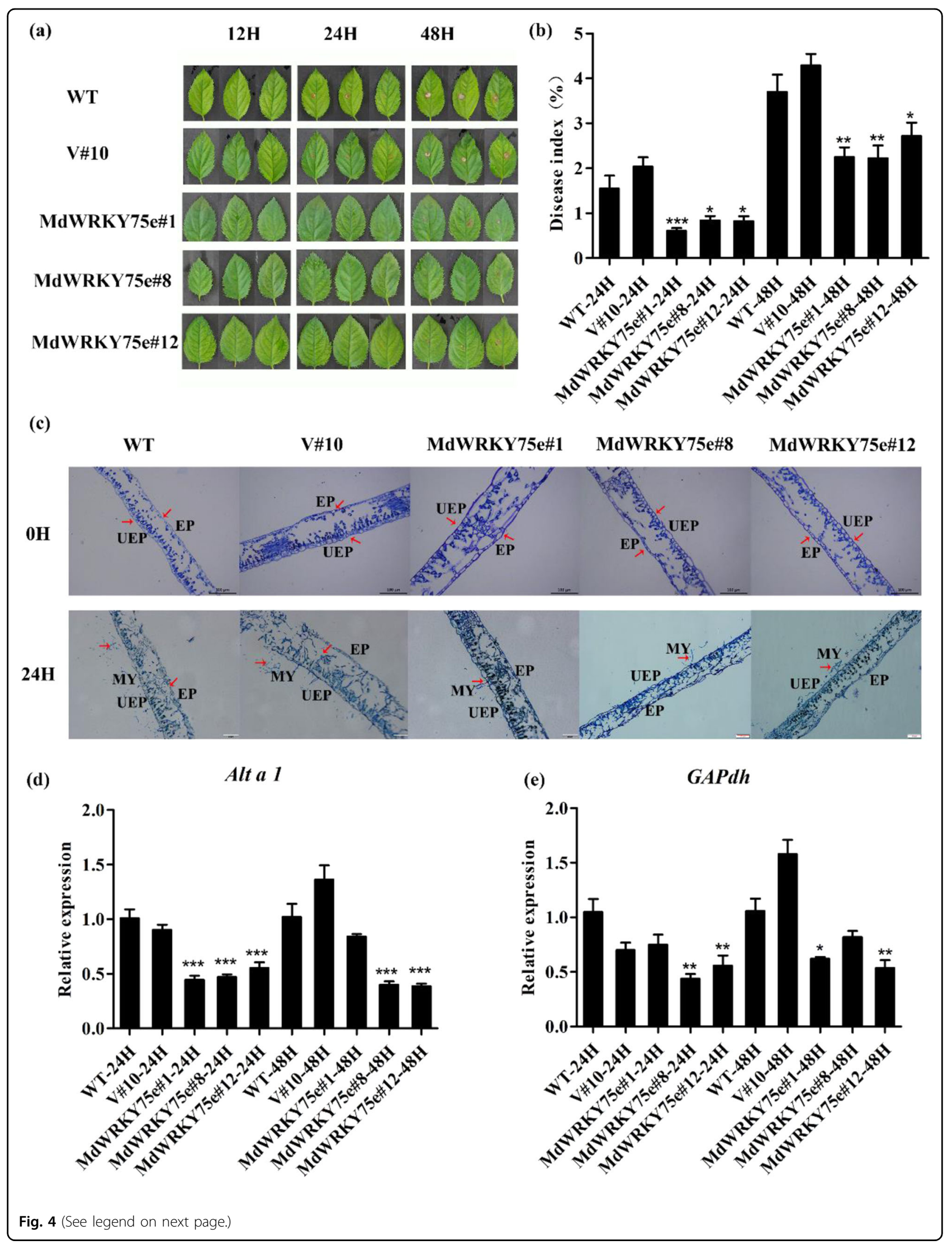


(see figure on previous page)

Fig. 4 Overexpression of MdWRKY75e enhanced A. alternata infection in transgenic apple. a Time course of A. alternata infection of WT, V\#10, and MdWRKY75e transgenic lines (\#1, \#8, and \#12) for $48 \mathrm{~h}$ for phenotype analysis. b Time course of $A$. alternata infection of WT, V\#10, and MdWRKY75e transgenic lines for $48 \mathrm{~h}$ for disease index analysis. c Phenotypes of paraffin sections of leaves infected with $\mathrm{A}$. alternata at 0 and $24 \mathrm{~h}$ in WT, V\#10, and MdWRKY75e transgenic lines. UEP upper epidermis, EP epidermis, and MY mycelium. d, e Time course of the expression of Alta1 (d) and GAPdh (e) genes in WT, V\#10, and MdWRKY75e transgenic lines under A. alternata infection for $48 \mathrm{~h}$. GAPdh and Alta1 in the internal transcribed spacer region sequences of Alternaria species can be used as marker genes for the severity of $A$. alternata apple pathotype infection ${ }^{40}$. WT: wild-type; V\#10: empty vector; and MdWRKY75e\#1, MdWRKY75e\#8, and MdWRKY75e\#12: MdWRKY75e transgenic lines

transfected apples with the pTRV2 system (Supplementary Fig. S5a). Semiquantitative RT-PCR was used to identify the efficiency of gene silencing and showed that the expression of MdWRKY75e was extremely low (Fig. 6a). Transcript analysis of young leaves further revealed that the transcription of MdWRKY75e, MdLAC7, and MdWFK1 was inhibited in the silenced plants (Fig. 6b-d); however, the expression of MdPR 9 and MdRFK1 did not change notably in the silenced plants (Supplementary Fig. S5b, c). We selected six MdWRKY genes that have close evolutionary relationships with MdWRKY75e to verify whether these gene expression levels were affected in MdWRKY75e knockdown lines. Our results showed that the expression levels of these genes were not significantly different in MdWRKY75e knockdown lines (Supplementary Fig. S5d-i). The above results indicated that the MdWRKY75e gene was silenced and did not affect the expression of other $M d W R K Y s$. We next compared the levels of disease resistance in leaves of in vitro silenced plants $48 \mathrm{~h}$ after infection with pathogens and found slight differences in pTRV2MdWRKY75e leaves compared with WT, empty vector (pTRV2), and pTRV2-GFP leaves (Fig. 6e). Time-course analyses of the disease index and GAPdh expression demonstrated greater disease susceptibility in pTRV2MdWRKY75e leaves than in WT, empty vector (pTRV2), and pTRV2-GFP leaves. Time-course analysis of Alta1 expression did not reveal remarkable changes in pTRV2$M d W R K Y 75 e$ leaves when compared with WT, empty vector (pTRV2), and pTRV2-GFP leaves (Fig. 6f-h). Similarly, laccase activity and lignin content were not significantly different in pTRV2-MdWRKY75e leaves, although these levels were somewhat lower than those in WT, empty vector (pTRV2), and pTRV2-GFP leaves (Fig. 6i, j). These findings suggested that silencing MdWRKY75e by VIGS increased $A$. alternata susceptibility in GL-3 transgenic plants.

\section{Phenotype and endogenous hormone contents in transgenic apple lines overexpressing MdWRKY75e}

The three GL-3 transgenic lines (\#1, \#8, and \#12) exhibited dwarfism, uniform serration of the leaf edges, a reduced leaf shape index, and a greener leaf color after 2 months of transplantation (Supplementary Figs. S4C and S6A, B).

SA and JA levels were quantitatively measured in transgenic plants. As shown in Supplementary Fig. S7A, B, JA and methyl jasmonate levels were slightly higher, whereas SA and methyl salicylate levels were remarkably lower in the transgenic lines than in the WT and empty vector lines. Moreover, administration of exogenous $0.1 \mathrm{mM} \mathrm{MeJA}$ and SA to WT, empty vector, and transgenic lines revealed that MeJA treatment significantly increased the expression of $M d M Y C 2$ in transgenic lines compared to that in WT and empty vector lines, although the level of MdNPR3 transcripts in transgenic lines did not change. SA treatment marginally increased the level of MdNPR3 transcripts in transgenic lines compared to that in WT and empty vector lines but did not affect $M d M Y C 2$ expression.

\section{Transgenic lines accumulate lower ROS and display higher antioxidant enzyme activities after infection with pathogens}

Histochemical staining with nitro blue tetrazolium (NBT) and diaminobenzidine (DAB) showed the levels of $\mathrm{O}_{2}{ }^{-}$and $\mathrm{H}_{2} \mathrm{O}_{2}$, respectively, in the leaves of plants $24 \mathrm{~h}$ after infection with pathogens. As shown in Fig. 7a, b, the NBT staining intensity in WT and empty vector leaves was stronger than that in transgenic leaves. DAB staining revealed evenly distributed brown precipitates all over the leaves of WT and empty vector plants, whereas the leaves of the transgenic plants were only lightly stained, indicating lower $\mathrm{O}_{2}^{-}$and $\mathrm{H}_{2} \mathrm{O}_{2}$ generation after infection with pathogens. Quantitative measurements further showed that the $\mathrm{H}_{2} \mathrm{O}_{2}$ and $\mathrm{O}_{2}{ }^{-}$ contents in the GL-3 transgenic lines were particularly lower than those in the WT and empty vector lines (Fig. 7c, d). Thus, histochemical staining and quantitative determinations demonstrated that the transgenic plants were more resistant to oxidative stress.

Furthermore, we examined the activities of four enzymes, SOD, CAT, POD, and PAL, as well as the MDA content in WT, empty vector, and transgenic lines after infection with pathogens. Interestingly, the enzyme activities of POD, CAT, and PAL were slightly higher in GL-3 transgenic lines than in WT and empty vector lines under normal conditions. Infection with pathogens significantly increased the activities of these enzymes in transgenic plants compared to those in WT and empty vector plants. In contrast, the enzyme activity of SOD and the MDA content in GL-3 transgenic lines under normal conditions were not significantly different from those after infection with pathogens, consistent with the results 


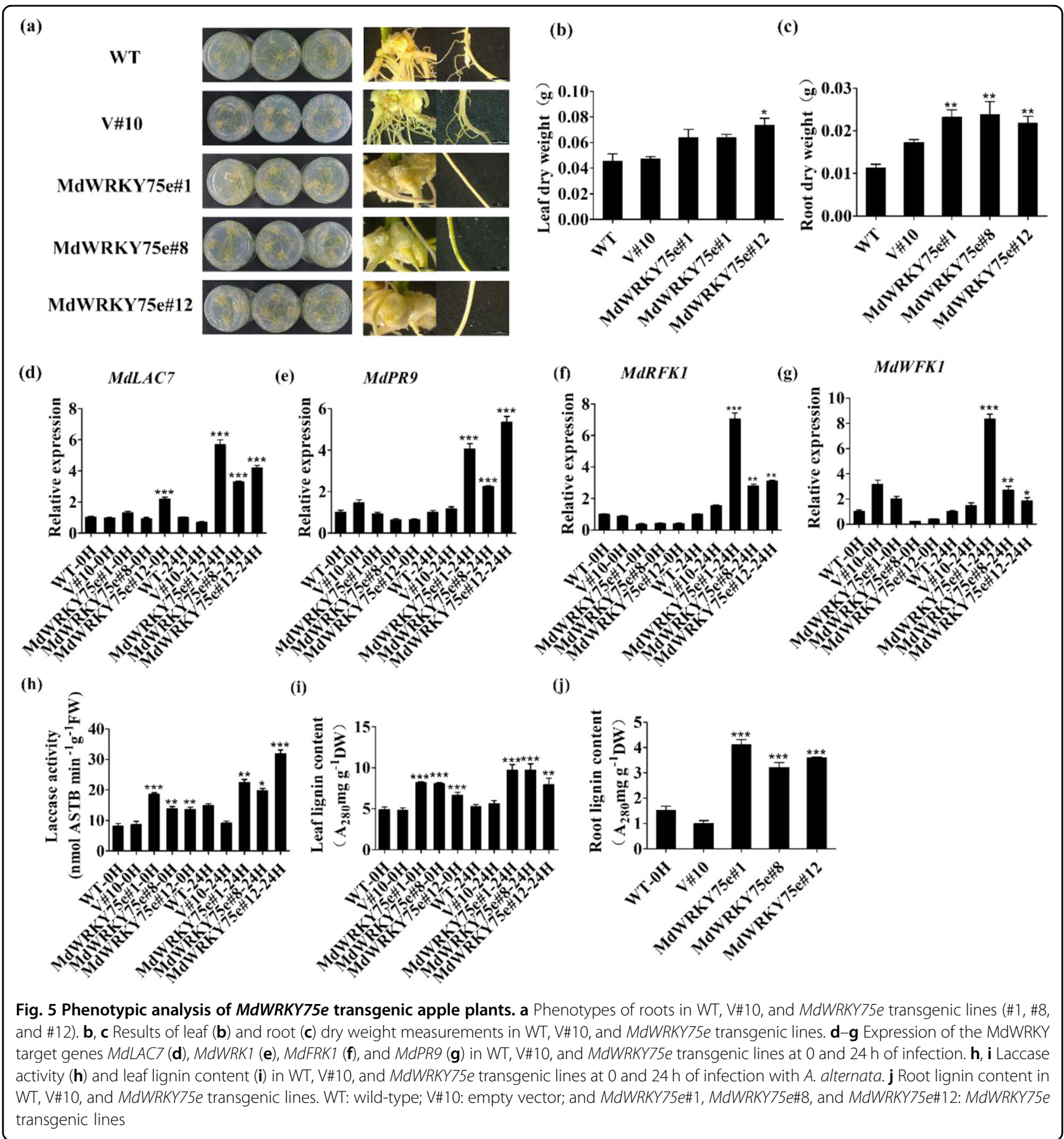

obtained in transgenic tobacco. Furthermore, we examined the expression of $N t S O D / M d S O D, N t C A T / M d C A T$, $N t P O D / M d P O D$, and NtPAL/MdPAL in tobacco and GL3 transgenic lines and found that except for NtSOD/ $M d S O D$, the transcription levels of the other genes were significantly higher in the transgenic plants, both before and after infection with pathogens; however, these transcription levels did not change remarkably between WT and empty vectors before and after pathogen infection treatment. Notably, these transcription levels were remarkably higher in the transgenic plants than in the WT and empty vector plants (Figs. 8 and 9).

Expression of genes involved in disease resistance responses in WT, empty vector, and transgenic lines before and after pathogen infection

The expression patterns of some genes involved in disease resistance responses were analyzed by qRT-PCR. 


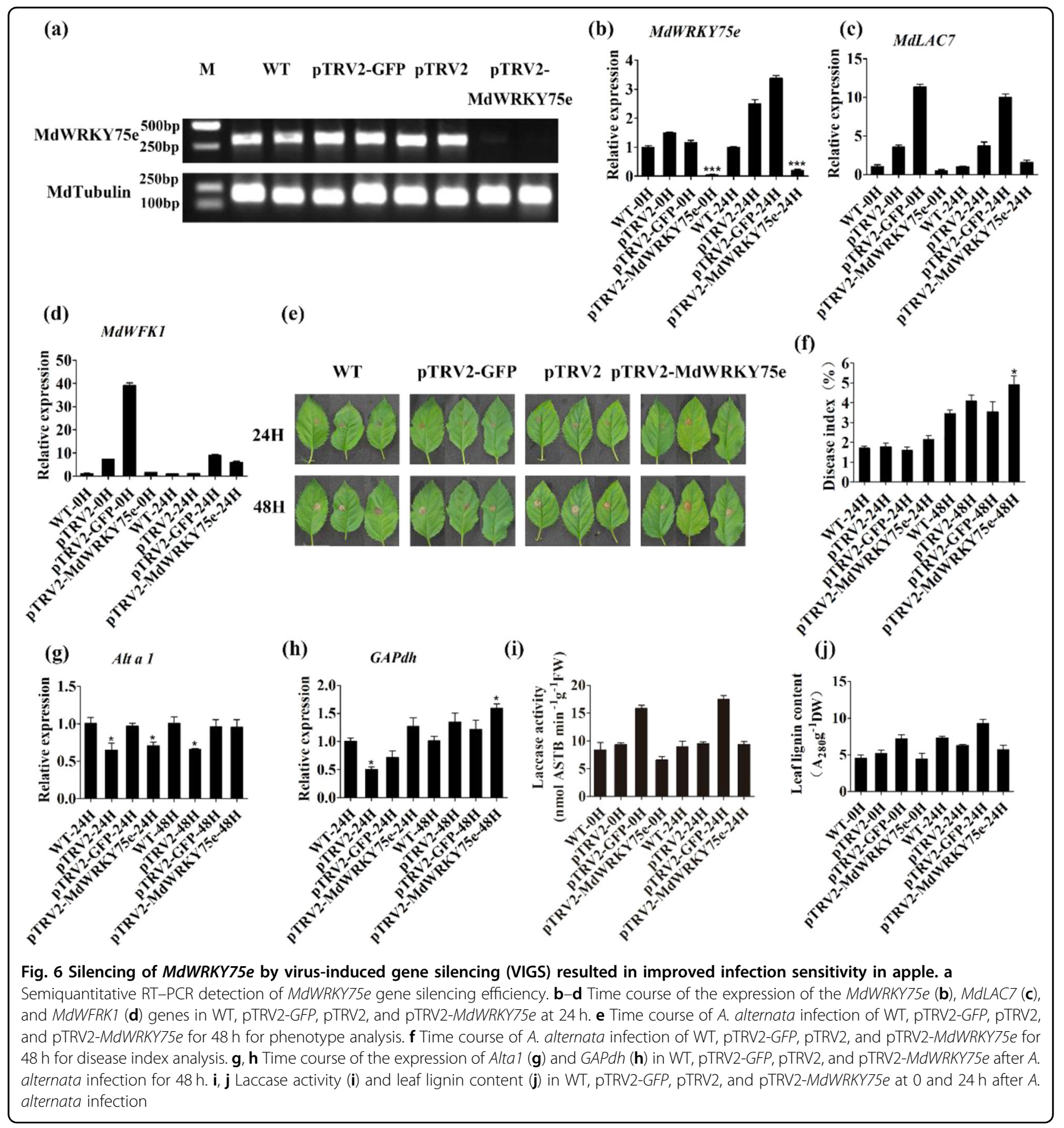

Under normal conditions, the expression levels of the genes did not change in transgenic tobacco lines (\#9, \#32, and \#36) and GL-3 transgenic lines (\#1, \#8, and \#12) compared to those in the WT and empty vector lines. The levels of NtPR5, NtHSR201, NtHSR515, NtACX1, NtJAR1, and Ntthil2 transcripts were slightly higher in the transgenic lines than in the WT and empty vector lines after infection with pathogens. In particular, NtNPR1 and $N t P R 1 b$ expression was remarkably increased in the transgenic line compared to that in the WT and empty vector lines. However, the expression levels of the NtPR1 $a / c$ and $N t S A-2$ genes in the transgenic plants were not notably different in comparison to those in the WT and empty vector plants (Fig. 10). Similarly, the MdPR1, MdPR2, Mdchit1, MdMYC2, and MdPDF1 transcript levels were particularly higher in the transgenic lines than in the WT and empty vector lines after infection with pathogens. Additionally, the expression levels of MdPR5, 


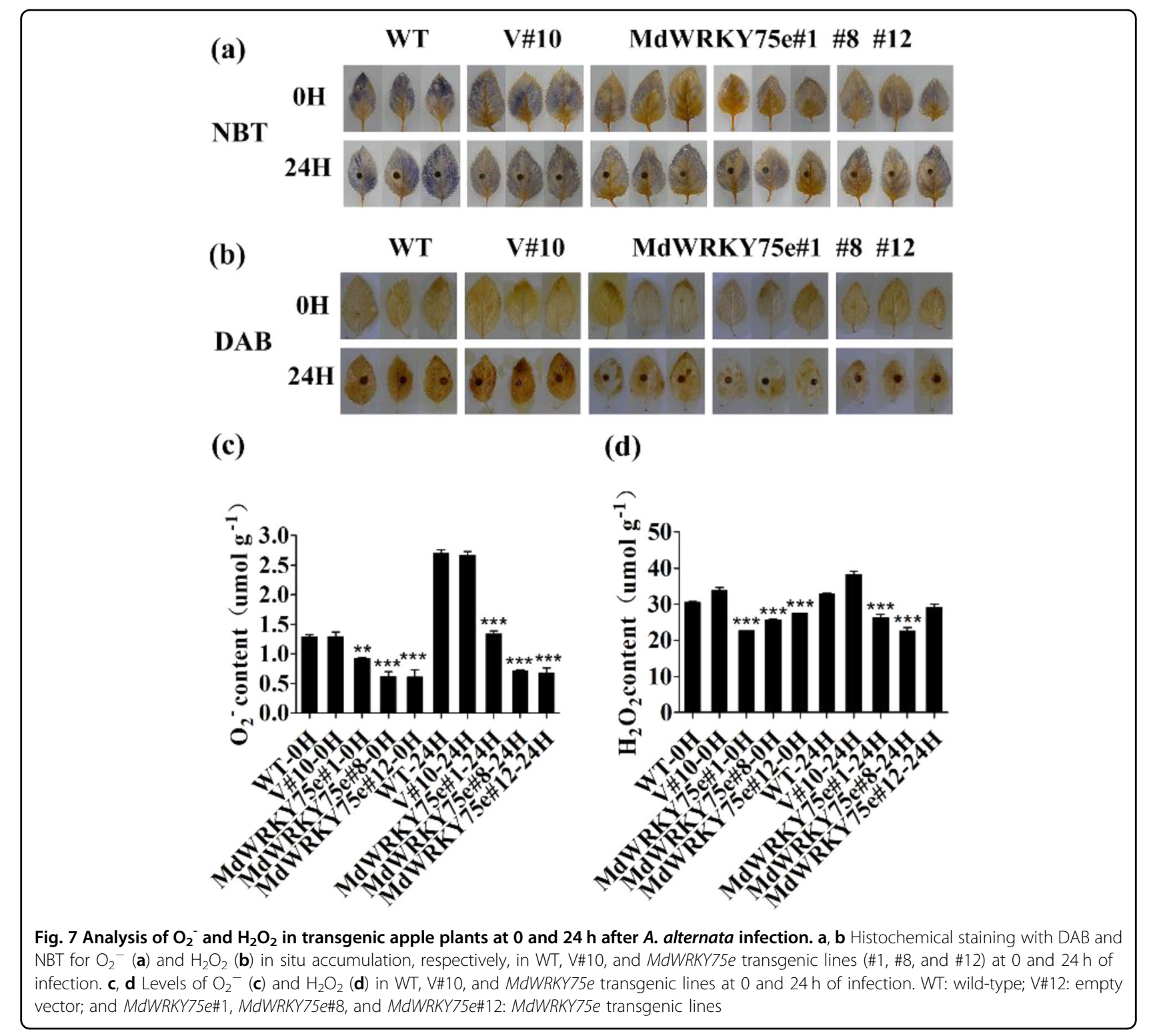

MdJAR4, and MdJA were slightly higher in the transgenic plants than in the WT and empty vector plants. Nevertheless, the level of MdNPR3 transcripts in the transgenic plants did not change compared to that in the WT and empty vector plants (Fig. 11).

\section{MdWRKY75e directly interacts with the promoter of MdLAC7}

Bioinformatics analysis indicated that the promoter sequences of $M d L A C 7, M d W R K 1, M d F R K 1$, and MdPR9, with sizes of $1221,1318,1977$, and $1530 \mathrm{bp}$, respectively, contained potential W-box (TTGACC) elements in their upstream regions (Supplementary Table S3). The upstream transcription start sites of the promoter sequences of $M d L A C 7, M d W R K 1, M d F R K 1$, and $M d P R 9$ were cloned into the reporter vector pHIS2 as bait, while
MdWRKY75e was used as prey (Supplementary Fig. S8a). First, self-activating reporter vectors were filtered by the concentration of 3-AT, which only inhibited pHIS2LAC7-, MdWRK1-, and MdPR9-transformed Y187 yeast cell growth at $30 \mathrm{mM}$. However, pHIS2-MdFRK1 selfactivation was not inhibited by $100 \mathrm{mM}$ 3-AT (Supplementary Fig. S8b). The vectors cotransformed with bait (pHIS2-LAC7, MdWRK1, and MdPR9) and prey (MdWRKY75e) and negative control yeast cells grew normally in the selection medium. However, the growth of yeast cells in the negative control was completely suppressed by the addition of $30 \mathrm{mM} 3-\mathrm{AT}$, while that in the positive control and bait (pHIS2-LAC7) and prey (MdWRKY75e) vector-containing cells were not (Fig. 12a, b). EMSA showed that the formation of a protein-DNA complex was observed when the 


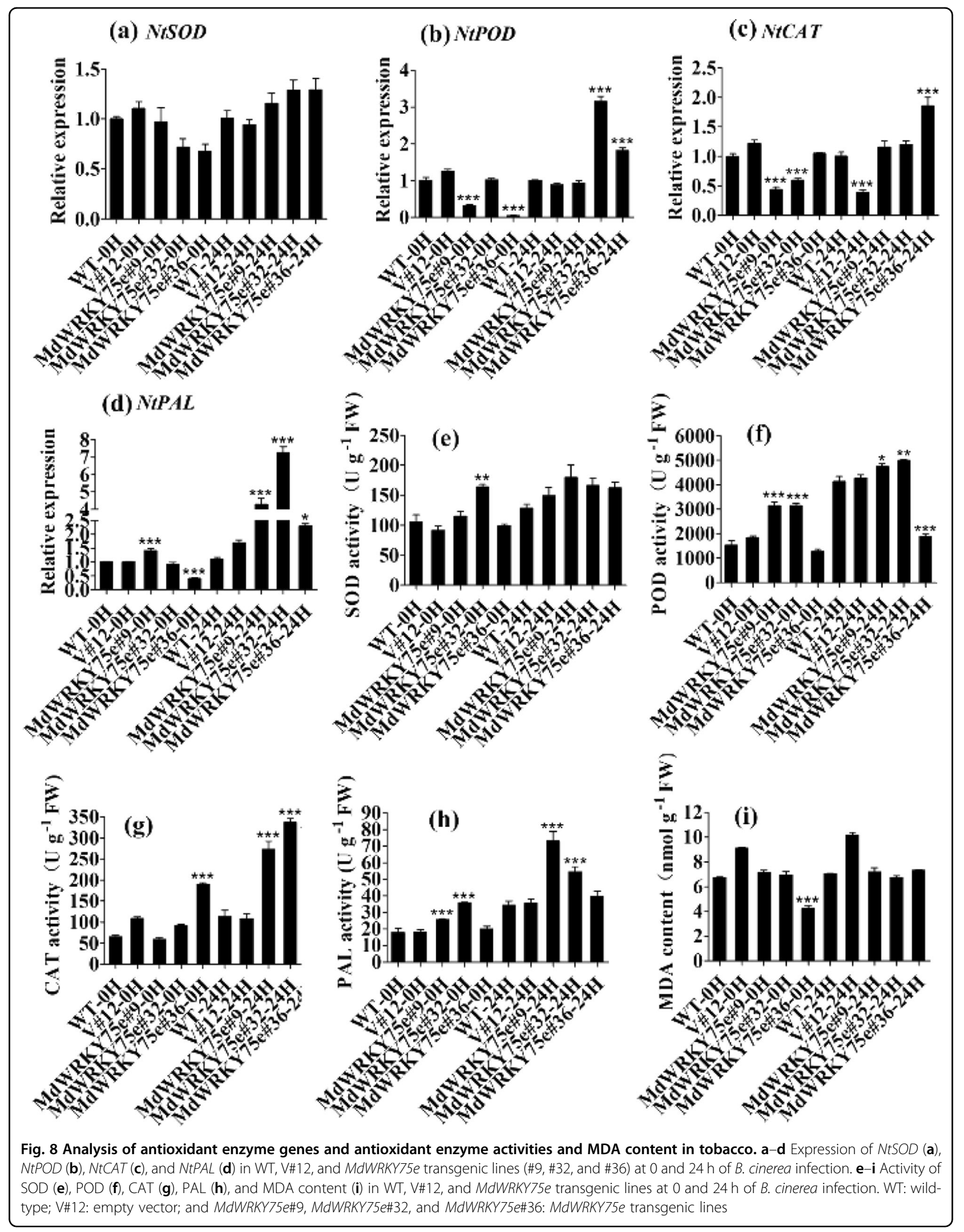




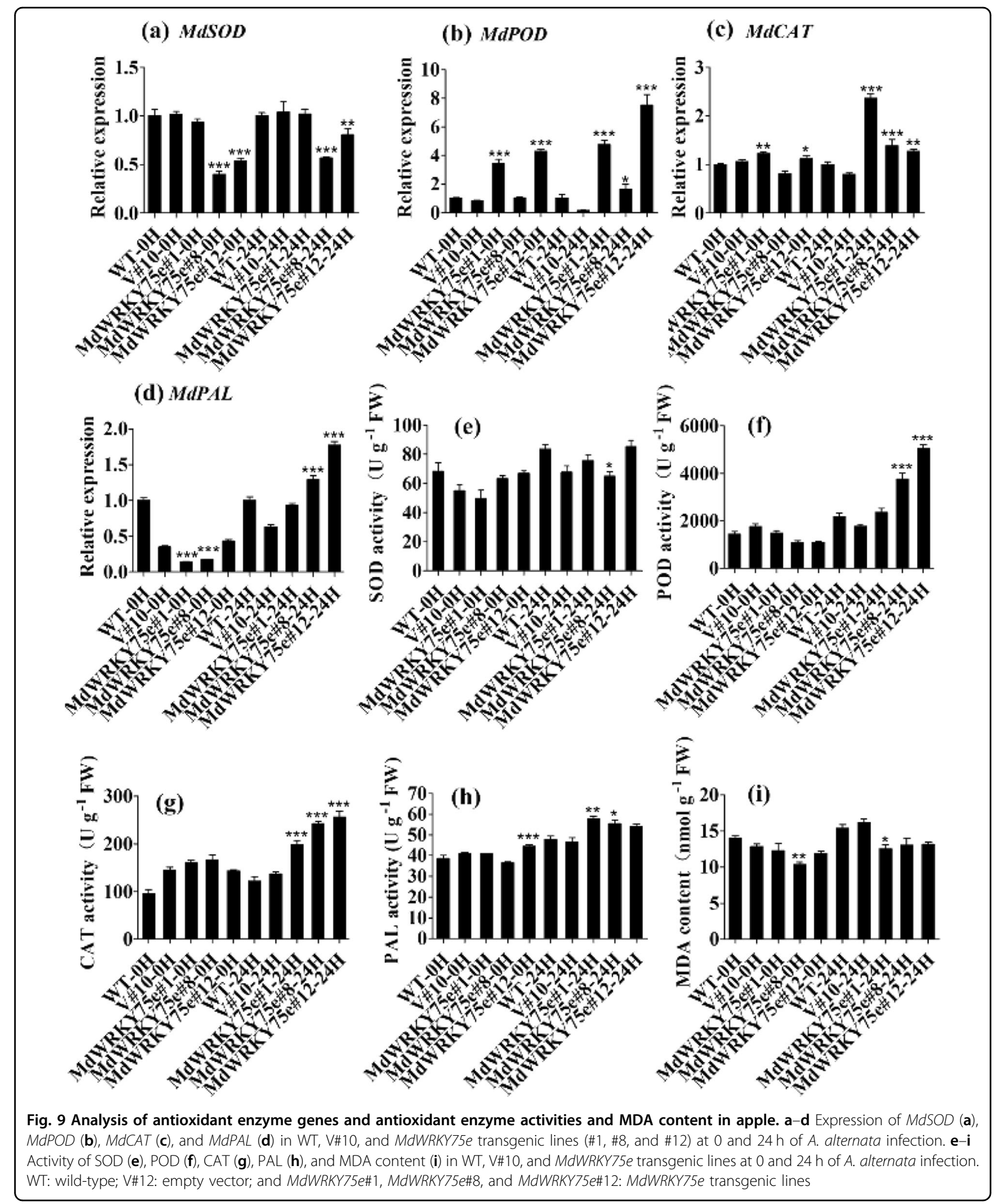

MdWRKY75e-MBP fusion protein was incubated with the probe containing the W-box element, whereas the complex was inhibited by the competitor probe. In addition, mutation of the W-box element in the probe was completely abolished (Fig. 12c). These results suggest that MdWRKY75e activates MdLAC7 promoter 


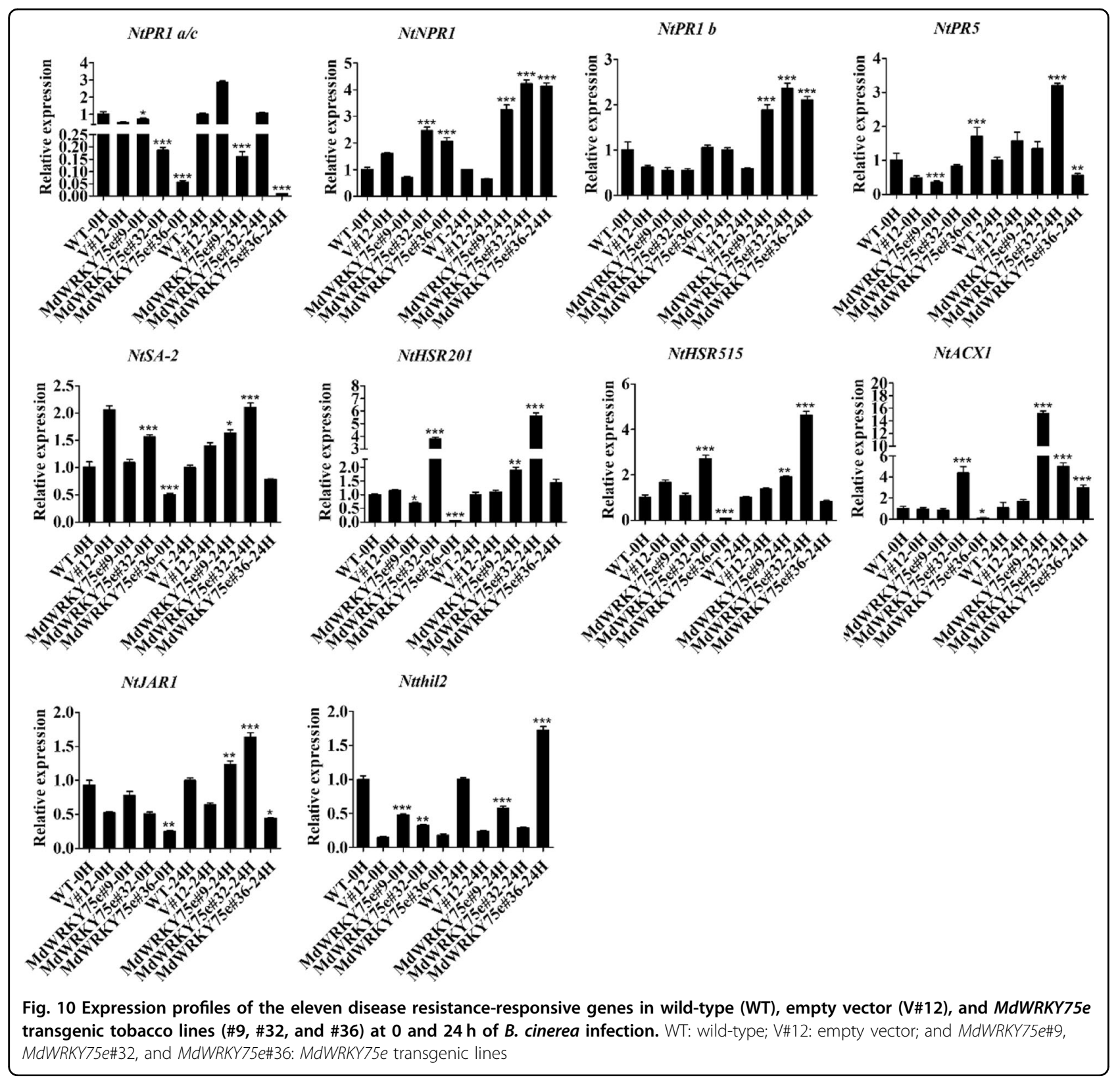

transcription. Transient expression tests were conducted for the interaction between MdWRKY75e and the promoter of $M d L A C 7$. Transient expression experiments for transforming the LUC reporter gene into tobacco leaves indicated that the LUC/REN ratio was considerably higher in leaves transformed with effectors and reporters than in controls (Supplementary Fig. S8c, d and Fig. 12d). Taken together, MdWRKY75e serves as a transactivator of MdLAC7.

\section{Discussion}

Plant WRKY proteins reportedly play a pivotal role in physiological and biological processes, such as hormonal signaling, carbohydrate synthesis, senescence, development, and secondary metabolite synthesis ${ }^{26,41}$. The WRKY TF superfamily consists of 72 and 119 members in Arabidopsis (Arabidopsis thaliana) and apple (M. domestica), respectively $^{42,43}$. A. thaliana AtWRKY75 and Fragaria ananassa Fa WRKY1 (AtWRKY75 homologous gene) play a pivotal role in modulating biological stress responses ${ }^{27,29,44}$. Conversely, FaWRKY1 negatively regulates resistance to the phytopathogenic fungus Colletotrichum acutatum in strawberry fruit ${ }^{45}$. Herein, we identified the WRKY TF $M d W R K Y 75 e$ (Group IIc) in M. domestica and demonstrated that its overexpression enhanced tolerance to infection with pathogens. We further showed that MdWRKY75e 


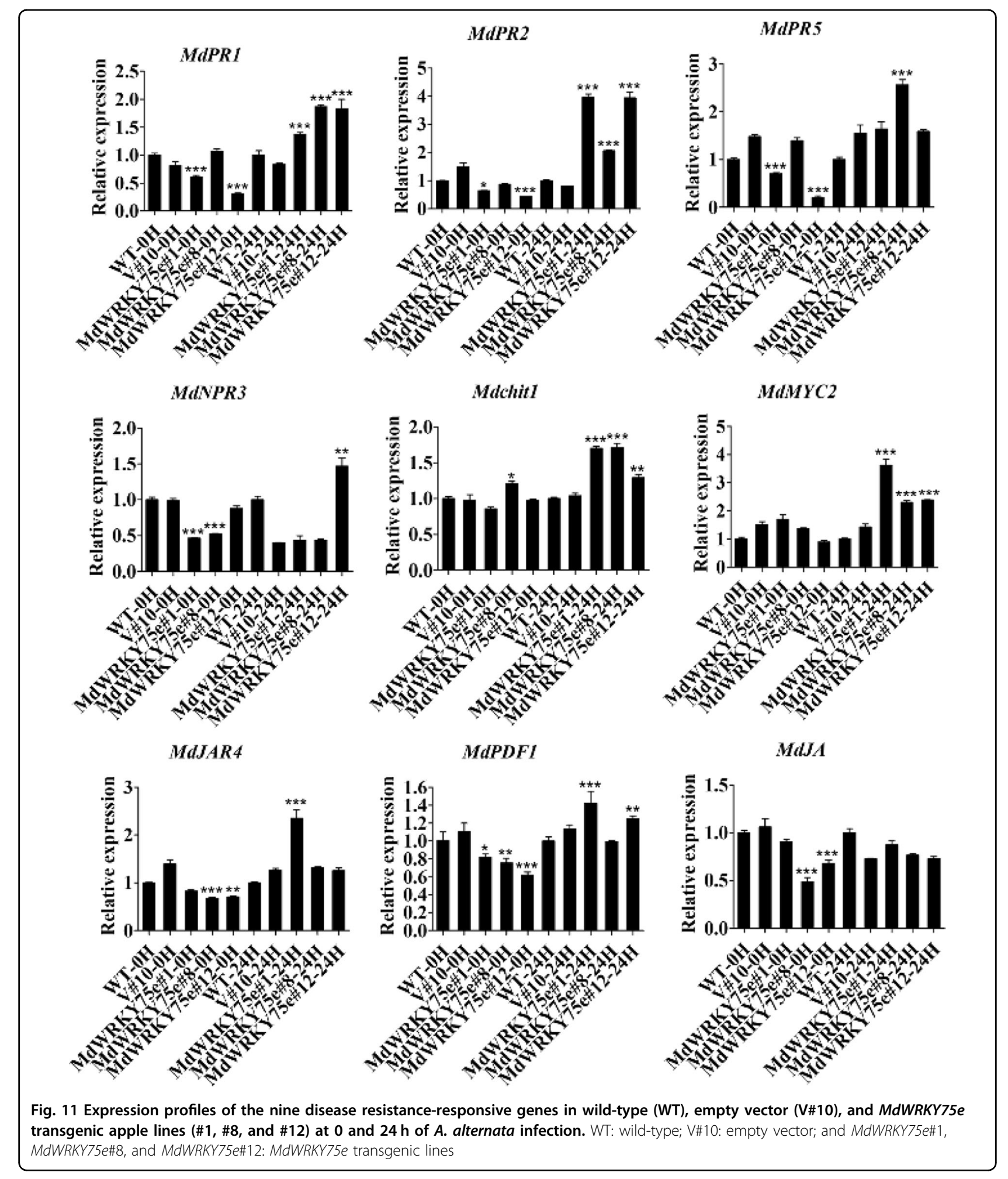

is a positive regulator of $M d L A C 7$ expression as well as laccase and lignin biosynthesis. Therefore, this study uncovered the molecular mechanism of MdWRKY75e and connected the function of WRKY to laccase and lignin biosynthesis to strengthen disease resistance.
Overexpression of WRKY genes in model or nonmodel plants reportedly induces tolerance to biotic stresses $^{27-29,33,34,44,46,47}$, suggesting that WRKY genes may have a high potential for disease resistance. Here, $M d W R K Y 75 d$ and MdWRKY75e transcript levels were 
(a)

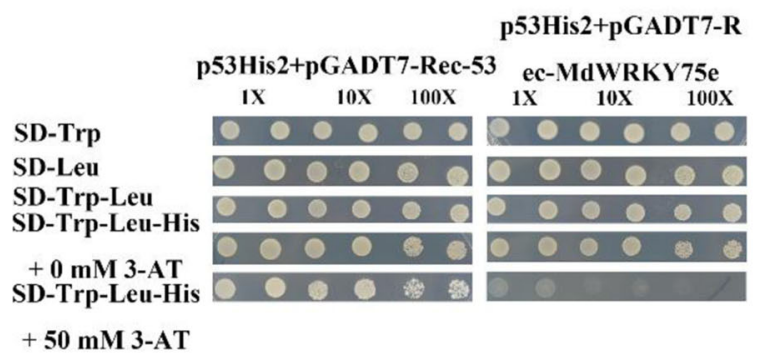

(c) (b)

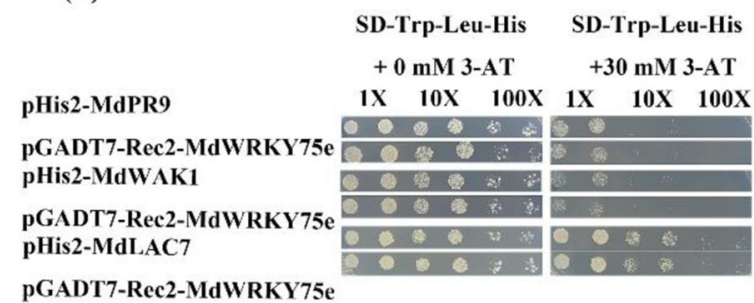

(d)

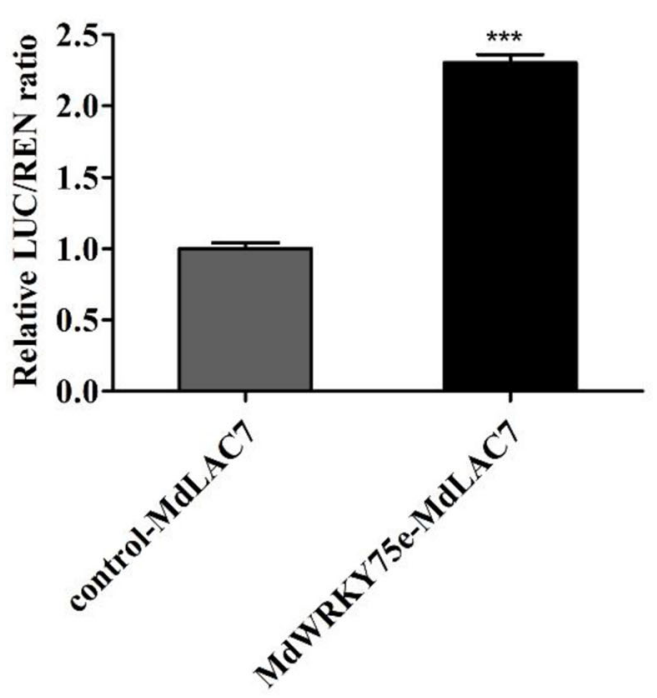

Fig. 12 MdWRKY75e associates with the promoter of MdLAC7 and initiates its expression. a Positive and negative constructs were utilized for the yeast one-hybrid experiment. b Prey and bait vectors were utilized for the yeast one-hybrid test. $\mathbf{c}$ The MdWRKY75e-MBP fusion protein directly bound to the W-box elements of the MdLAC7 promoters. - absence, + presence. $\mathbf{d}$ Real-time expression experiment of the activity of promoters coconverted with the MdWRKY75e effector and MdLAC7 reporter in tobacco leaves

markedly increased by A. alternata infection. Analysis of the tissue-specific expression of MdWRKY75d and $M d W R K Y 75 e$ showed that these genes were mainly involved in fruit development and leaf development, respectively. Transgenic lines overexpressing MdWRKY75e displayed better disease resistance to infection with pathogens such as $B$. cinerea and $A$. alternata. However, MdWRKY75d transgenic lines displayed poor resistance to infection with pathogens such as $B$. cinerea. MdWRKY75e knockdown rendered apple slightly susceptible to $A$. alternata, indicating that MdWRKY75e is a positive regulator of disease resistance.

ROS accumulation in biological systems relies on the homeostasis between the formation and removal of ROS under physiological conditions ${ }^{19,20}$. We evaluated ROS levels in transgenic and WT apples. Histochemical staining with NBT and DAB revealed lower contents of $\mathrm{H}_{2} \mathrm{O}_{2}$ and $\mathrm{O}_{2}{ }^{-}$in the transgenic lines than in the WT and empty vector lines after infection with pathogens. Plant cells have a sophisticated antioxidant defensive system for ROS removal, which is accomplished by ROS scavenging enzymes, such as POD, CAT, and $\mathrm{SOD}^{20}$. The activities of CAT and POD were observed to be much higher in transgenic tobacco and GL-3 plants than in their respective WT and empty vector lines after infection with pathogens. However, SOD activities and the MDA content did not change in tobacco and GL-3 transgenic plants. These genes are also involved in the biosynthesis of antioxidant enzymes (NtSOD/MdSOD, NtCAT/ $M d C A T$, and NtPOD/MdPOD). qRT-PCR analysis revealed that the expression levels of these genes, except for $N t S O D / M d S O D$, were higher in the transgenic plants than in the WT and empty vector plants after infection with pathogens, indicating that MdWRKY75e overexpression effectively induces a detoxification system to remove ROS generated during infection with pathogens.

The pathogenesis-related genes $P R-1$ and $P R-2(\beta-1,3-$ glucanase), the SA-responsive marker gene $N P R 1$, and 


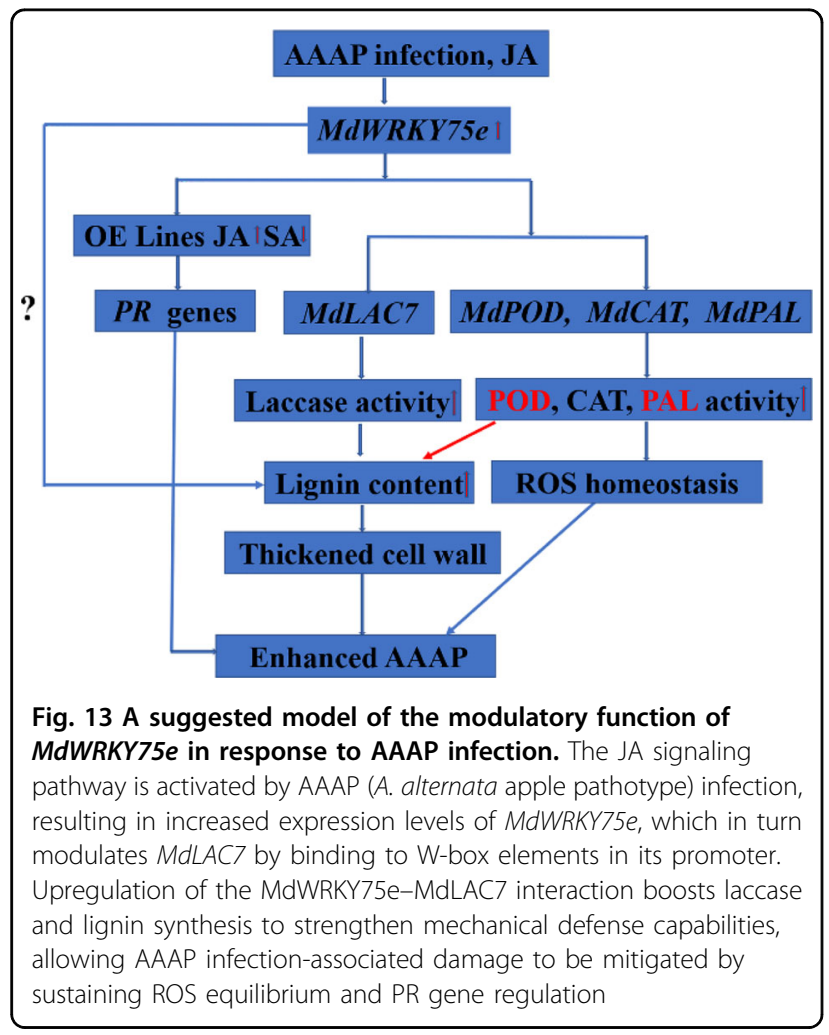

PR-5 (thaumatin-like proteins) are often activated by SA to mediate plant defense responses ${ }^{48,49}$. Similarly, MYC2, $J A Z$, Chit1, and the JA-responsive fungal pathogen marker genes Thi2.1 and PDF1.2 are often activated by JA to mediate plant defense responses ${ }^{17,18,50,51}$. Liu et al. ${ }^{52}$ found that NtPR1a overexpression may be mainly dependent on the hypersensitive response (HR)-associated genes NtHSR201, NtHSR515, and NtHIN1; the SAassociated genes NtPR1 and NtPR2; and the JA-associated gene $N t P R 1 b$ to enhance resistance to $R$. solanacearum in tobacco. Our results showed that NtNPR1, NtPR1b, MdPR1, MdPR2, Mdchit1, MdMYC2, and MdPDF1 transcript levels were remarkably upregulated in transgenic plants compared with those in WT and empty vector plants. NtPR5, NtHSR201, NtHSR515, NtACX1, NtJAR1, Ntthil2, MdPR5, MdJAR4, and MdJA transcript levels were slightly higher in transgenic plants than in WT and empty vector plants, both before and after infection with pathogens. However, NtPR1 a/c, NtSA-2, and $M d N P R 3$ expression levels hardly changed in the transgenic plants in comparison to those in the WT and empty vector plants (Figs. 10 and 11). More importantly, WRKY62 and WRKY70 play an essential role in the crosstalk between the SA and JA pathways, acting as regulatory nodes of the JA and SA signaling pathways ${ }^{53,54}$. $M d W R K Y 75$ e transcript levels were markedly increased by MeJA treatment but suppressed by SA treatment. Furthermore, an investigation of endogenous hormones showed that JA and methyl jasmonate contents were higher in transgenic lines than in WT and empty vector lines. SA and methyl salicylate levels were remarkably lower in transgenic lines than in WT and empty vector lines. These results demonstrated that JA signaling pathways play key roles in the enhanced disease resistance of transgenic plants overexpressing MdWRKY75e, while SA signaling pathways play secondary roles in this regard.

PAL, peroxidases, and laccases have important roles in lignin synthesis in the phenylpropanoid pathway, which provides plants with a physical barrier against pathogens and pests ${ }^{2-24,55,56}$. Interestingly, the activities of PAL, POD, and laccases were significantly higher in transgenic plants than in WT and empty vector plants. Leaf and root dry weights were higher in transgenic plants than in WT and empty vector plants. The roots of transgenic MdWRKY75e plants had deeper color and increased lignification, and root hair formation was particularly suppressed in GL-3 transgenic lines; this was similar to observations of AtWRKY75 ${ }^{57,58}$. Notably, the lignin contents in the leaves and roots of MdWRKY75e transgenic plants were markedly higher than those of WT and empty vector plants. However, the laccase activity and lignin content were not significantly different, although they were slightly lower in transgenic lines than in the WT, empty vector (pTRV2), and pTRV2-GFP lines. MdWRKY9 overexpression conferred intensive dwarfing in the M26 rootstock of apple ${ }^{59}$. We further observed that three GL-3 transgenic lines (\#1, \#8, and \#12) exhibited dwarfism, uniform serration of the leaf edges, and a greener leaf color after 2 months of transplanting; these phenotypes indicate enhanced stress resistance.

At the transcriptional level, WRKY TFs can specifically regulate target gene expression by binding to the W-box (T)TGACC(A/T) in their promoters ${ }^{41}$. We examined the W-box elements of MdLAC7 (laccase 7), MdWRK1, $M d F R K 1$, and MdPR9 (POD). Interestingly, MdLAC7, $M d W R K 1, M d F R K 1$, and MdPR9 expression (especially $M d L A C 7)$ was intensely induced in MdWRKY75e-overexpressing GL-3 lines after infection with pathogens. Yeast one-hybrid, EMSA, and transient expression experiments supported the straightforward and particular interactions between MdWRKY75e and the MdLAC7 promoter. These data enabled us to speculate that $M d L A C 7$ is a target gene of MdWRKY75e, indicating that MdLAC7 may be regulated by WRKY TFs. Here, we present a model of the role of the modulatory function of MdWRKY75e during the response to infection with pathogens, which may directly regulate the synthesis of lignin (Fig. 13). Infection with pathogens induces the JA signaling pathway, resulting in elevated expression of $M d W R K Y 75$ e, which regulates $M d L A C 7$ by binding to W-box elements in its promoter. Upregulation of the MdWRKY75e-MdLAC7 interaction contributes to 
laccase and lignin synthesis, and ROS scavenging systems contribute to maintaining ROS homeostasis. We provide herein a new understanding of the molecular mechanisms of laccase and lignin synthesis and ROS removal in disease resistance.

In summary, we confirmed a disease resistance-responsive WRKY TF, MdWRKY75e, extracted from 'Sushuai' apple that serves as an active modulator of disease resistance. MdWRKY75e-overexpressing plants accumulated more laccase and lignin, which in turn stimulated downstream MdLAC7 expression. Establishment of the WRKY-LAC7 and ROS scavenging network offers useful knowledge of the function and potential molecular mechanism of WRKY and broadens our understanding of the complicated disease resistance signaling network. However, MdWRKY75e may also regulate other disease resistance-responsive genes, and further studies are needed to uncover other components associated with $M d W R K Y 75 e$ to gain deeper insight into the molecular mechanisms of $M d W R K Y 75 e$ function in disease resistance.

\section{Materials and methods}

Plant material, growth environment, and stress treatments

Nicotiana tabacum (K326), 'Sushuai' apple exhibiting greater disease resistance ${ }^{60}$, and transgenic (tobacco and GL-3) plants overexpressing MdWRKY75d and MdWRKY75e derived from 'Sushuai' apple were cultivated in Nanjing, China, under normal farming practices or grown in a greenhouse. Leaves were excised from $M$. domestica 'Sushuai' apple and transgenic lines (tobacco and GL-3) for infection with pathogens as well as $0.1 \mathrm{mM}$ $\mathrm{JA}$ and $0.1 \mathrm{mM}$ SA treatments. The leaves were cleaned and cultivated for $6 \mathrm{~h}$ to reduce mechanical damage, followed by exposure to appropriate treatments. N. tabacum (K326) and B. cinerea strain Bt56 preserved in the laboratory were used. All biological samples were stored at $-80^{\circ} \mathrm{C}$ until further analysis.

\section{RNA extraction and quantitative real-time PCR analysis}

Total RNA was derived from frozen leaves using an RNA extraction kit (Fuji, China) and treated with RNasefree DNase I (TaKaRa, Tokyo, Japan) to reduce potential redundant genomic DNA. First-strand cDNA was generated using a reverse transcription kit (TaKaRa, Tokyo, Japan) following the manufacturer's instructions. qRT-PCR was conducted with a SYBR-Green PCR kit (TakaRa, Tokyo, Japan) according to the manufacturer's instructions. Each sample was analyzed in four replicates, and the $2^{-\Delta \Delta \mathrm{Ct}}$ method $^{61}$ was used to compute the relative expression levels of each gene. Tubulin was used as the reference gene for $M$. domestica and tobacco to normalize gene expression levels. The primers were designed using primer-BLAST (https://www.ncbi.nlm.nih.gov/ tools/primer-blast) (Supplementary Table S1).

\section{MdWRKY75s cloning and sequence analysis}

The MdWRKY75 sequence (https://phytozome-next.jgi. doe.gov/info/Mdomestica_v1_1) was used as a template for designing gene-specific primers (Supplementary Table S2) to amplify MdWRKY75d and MdWRKY75e genes using RT-PCR. The PCR product was purified, subcloned into the clone007 blunt simple vector, and sequenced at TsingKe (Nanjing, China). Multiple alignments of the homologous amino acid sequence of MdWRKY75s from different species from the NCBI website were performed using Clustal W and BioXM. The phylogenetic tree was created in MEGA 7.0 software by the neighbor-joining method and bootstrap test using 1000 replicates. The molecular weight and theoretical isoelectric point were forecasted using the Expert Protein Analysis System (http://web.expasy.org/compute_pi/).

\section{Subcellular localization and transactivating activity of MdWRKY75s}

PCR amplification of the full-length cDNAs of MdWRKY75d and MdWRKY75e was conducted using primers (Supplementary Table S2) comprising NcoI and SpeI restriction sites, and then the cDNAs were ligated to the pCAMBIA1302 vector to produce a fusion construct (35S-MdWRKY75-GFP). After sequence identification, the control vector (pCAMBIA1302) and the fusion construct (35S-MdWRKY75-GFP) were transformed into Agrobacterium tumefaciens strain $\mathrm{AH} 105$ by heat shock. The 35S-MdWRKY75-GFP and free 35S-GFP plasmids were transiently coexpressed with a nuclear marker (35SD53-RFP vector) ${ }^{62}$.

For the transactivation assay, the CDSs of MdWRKY75d and MdWRKY75e were amplified by PCR using primers (Supplementary Table S2) comprising NcoI and BamHI restriction sites. The resultant fragments containing MdWRKY75d and MdWRKY75e were fused via recombination reactions downstream of the yeast GAL4 DNA binding domain of pGBKT7. The negative control (pGBKT7) and the fusion vector were separately expressed following the manufacturer's instructions in yeast strain AH109.

\section{Vector construction, plant transformation, and identification of transgenic lines}

The full-length coding regions of MdWRKY75d and MdWRKY75e were PCR-amplified and (Supplementary Table S2) inserted into the pCAMBIA1301 vector. The leaf disc transformation method was used to transform tobacco $\mathrm{K}_{326} 6^{63}$. Normal growth of transgenic tobacco was identified by cutting the leaves of resistant tobacco for GUS staining, followed by semiquantitative RT-PCR analysis and qRT-PCR of the gene-specific primers ${ }^{64}$.

The full-length coding region of MdWRKY75e was PCR-amplified (Supplementary Table S2) and inserted 
into the pCAMBIA2300 vector. Transgenic GL-3 apple plants were produced from leaf fragments by Agrobacterium-mediated transformation ${ }^{65}$. We performed GUS staining and semiquantitative RT-PCR analysis with isolated RNA to check for the presence of the transgene in putatively transformed lines. Overexpression of MWRKY75e was confirmed by qRT-PCR.

\section{Assessment of pathogen tolerance in transgenic lines}

The WT, empty vector, and transgenic lines were infected with pathogens to investigate their disease resistance capacities. For pathogen infection treatment, leaves were collected from 45-days-old seedlings of tobacco transgenic lines and 60-days-old seedlings of apple transgenic lines. The plant pathogens $B$. cinerea and $A$. alternata were grown on potato dextrose agar (PDA) media (Solarbio, Beijing, China) at $24^{\circ} \mathrm{C}$ for 8 days. Fifteen to twenty leaves of the WT, empty vector, and transgenic lines (tobacco and apple) were collected, washed with $\mathrm{ddH}_{2} \mathrm{O}$ three times, and cultured for $6 \mathrm{~h}$ for infection with pathogens. The leaves were infected using an approximately 3-mm diameter bacterial plaque acquired by a hole puncher to maintain consistency. Afterward, images were taken at various time points, and the leaf lesion areas were measured by ImageJ software ${ }^{65,66}$.

Samples of leaves and roots corresponding to the various time points were collected and frozen instantly in liquid nitrogen and stored at $-80^{\circ} \mathrm{C}$ for analyses of gene expression and enzyme activity. The MDA content, antioxidant enzyme activities, laccase activity, and lignin content in the leaves, and partial index in the roots at the corresponding time points were examined. Infection with pathogens was repeated at least three times. Three replicates were utilized for each line after the infection treatment.

\section{Promoter isolation and $\mathrm{Y} 1 \mathrm{H}$ assay}

Genomic DNA was extracted from apple leaves by applying a DNA extraction kit (TIANGEN). PCR amplification of the promoter sequences of MdPR9, MdRFK1, $M d W A K 1$, and $M d L A C 7$ was accomplished with primers (Supplementary Table S2) using genomic DNA as a template. The amplified products were subsequently subcloned into the clone007 blunt simple vector and then sequenced at TsingKe. Transcription start sites and conserved cis-element motifs of promoters were analyzed using the PLACE (http://www.dna.affrc.go.jp/htdocs/ PLACE/) and Plant-CARE (http://bioinformatics.psb. ugent.be/webtools/plantcare/html/) websites, respectively. For this purpose, full-length $M d W R K Y 75 e$ was RT-PCR-amplified using primers (Supplementary Table S2) and incorporated into the EcoRI and XhoI sites of pGADT7-Rec to produce the effector vector pGADT7Rec-MdWRKY75e. Simultaneously, promoter sequences of MdPR9, MdRFK1, MdWAK1, and MdLAC7 were amplified using primers (Supplementary Table S2) comprising the EcoRI and $S a c \mathrm{I}$ restriction sites and subcloned into $\mathrm{pHis} 2$ to generate the reporter vectors pHis2-MdPR9, pHis2-MdRFK1, pHis2-MdWAK1, and pHis2-MdLAC7, respectively. A yeast one-hybrid experiment was conducted using the Matchmaker Gold Yeast One-Hybrid Library Screening System (Clontech, USA). Y187 yeast cells cotransformed with effector vector and reporter vector were cultured at $28{ }^{\circ} \mathrm{C}$ for 3 days on SD-Trp-LeuHis medium supplemented with or without $30 \mathrm{mM} 3-\mathrm{AT}$.

\section{Electrophoretic mobility shift assay}

Electrophoretic mobility shift assay (EMSA) was performed using an EMSA Probe Biotin Labeling Kit and a Chemiluminescent EMSA Kit (Beyotime Biotechnology, Shanghai, China). The CDS of the MdWRKY75e gene was cloned into the pMAL-c5X vector, which was then transformed into Rosetta (DE3) cells for the subsequent production of the MdWRKY75e-MBP fusion protein. Probes specific for the promoter fragments and their mutants (Supplementary Table S2) were synthesized by Sangon Biotechnology Co., Ltd. (Shanghai, China). In $M d L A C 7$, the $5^{\prime}$-TTGACC- $3^{\prime}$ motif was replaced by $5^{\prime}$ TCGAAC- $3^{\prime}$ in the mutant probe. EMSAs were performed according to the manufacturer's protocol and as described $^{67}$.

\section{Transient expression assay}

VIGS-mediated inhibition of MdWRKY75e was conducted as described previously ${ }^{68,69}$. To construct the pTRV2-MdWRKY75e vector, a $180 \mathrm{bp}$ fragment of the MdWRKY75e CDS (24-204 bp) was inserted into the EcoRI and XhoI sites of tobacco rattle virus-based vector 2 (pTRV2) to produce the pTRV2-MdWRKY75e vector. Empty pTRV2 and pTRV2-GFP constructs were utilized as controls.

Primers containing SacI and BamHI restriction sites were used to amplify the coding region of MdWRKY75e (Supplementary Table S2), which was inserted into pCAMBIA1301 to yield an effector plasmid. MdPR9, MdRFK1, $M d W A K 1$, and MdLAC7 promoter sequences were amplified with specific primers (Supplementary Table S2) containing HindIII and BamHI restriction sites and inserted into the pGreen II 0800-LUC reporter vector ${ }^{70}$. The effector and reporter vectors were transfected into A. tumefaciens GV3101 cells. Transient expression experiments were conducted in tobacco as described previously $y^{71,72}$ with minor modifications. The analysis of the LUC/REN ratio was performed as described previously ${ }^{73}$.

\section{Histochemical staining and physiological measurement}

GUS staining was conducted as described previously ${ }^{31}$. Histochemical staining for $\mathrm{H}_{2} \mathrm{O}_{2}$ and $\mathrm{O}_{2}{ }^{-}$accumulation was carried out with DAB and NBT, respectively ${ }^{74,75}$. 
$\mathrm{H}_{2} \mathrm{O}_{2}$ and $\mathrm{O}_{2}{ }^{-}$levels were measured using analytical kits (Lai Er Bio-Tech, HeFei, China). Toluidine blue O staining of paraffin-embedded sections was performed as described previously ${ }^{65,76}$.

Analysis of the MDA content, SOD, POD, CAT, and PAL enzyme activities, laccase activity, and lignin content levels was performed using analytical kits (Lai Er BioTech, HeFei, China).

\section{Measurement of SA and JA contents}

Quantitative measurement of SA and JA contents was performed using an Agilent 1290/AB Qtrap6500 HPLC-MS/MS Analysis system (Agilent, Santa Clara, CA, USA) following the manufacturer's protocol, as described previously ${ }^{77,78}$.

\section{Statistical analysis}

The experiments were repeated at least three times for each stress treatment. For a representative experiment with at least three independent replicates, data are expressed as the mean \pm SE. Data were analyzed by Tukey's multiple range tests in the ANOVA program of SPSS (IBM SPSS 22, Chicago, IL, USA). ${ }^{*} P<0.05,{ }^{* *} P<$ 0.01 , and ${ }^{* * *} P<0.001$ were considered significant.

\section{Acknowledgements}

This work was supported by the National Natural Science Foundation of China (grant number 31872074), the National Key R\&D Program of China (2019YFD1000100), and Priority Academic Program Development of Jiangsu Higher Education Institutions. We thank Dr. Zhihong Zhang, Shenyang Agricultural University, for providing tissue-cultured GL-3 plants and Yunzhong Guo, Northwest A\&F University, for providing the A. alternata.

\section{Author details}

${ }^{1}$ College of Horticulture, Nanjing Agricultural University, Nanjing, People's Republic of China. ${ }^{2}$ College of Agriculture, Nanjing Agricultural University, Nanjing, People's Republic of China. ${ }^{3}$ Institute of Botany, Jiangsu Province and Chinese Academy of Sciences (Nanjing Botanical Garden, Memorial Sun Yatsen), Nanjing, People's Republic of China

\section{Author contributions}

S.Q. and S.W. designed the research. Y.H. performed the experiments. S.Q., Y.H., and W.Z. wrote the manuscript. C.S. helped to conduct EMSA. X.Y. and W.C. provided technical support. L.C. and T.Z. helped analyze the data.

\section{Data availability}

The data used to support the findings of this study are available from the corresponding author upon reasonable request.

\section{Competing interests}

The authors declare no competing interests.

Supplementary information The online version contains supplementary material available at https://doi.org/10.1038/s41438-021-00701-0.

Received: 27 April 2021 Revised: 8 August 2021 Accepted: 2 September 2021

Published online: 11 October 2021

\section{References}

1. Harteveld, D. O. C., Akinsanmi, O. A., Dullahide, S. \& Drenth, A. Sources and seasonal dynamics of Alternaria inoculum associated with leaf blotch and fruit spot of apples. Crop Prot. 59, 35-42 (2014).

2. Zhang, C. X., Tian, Y. \& Cong, P. H. Proteome analysis of pathogen-responsive proteins from apple leaves induced by the Alternaria Blotch Alternaria alternata. PLoS ONE 10, e0122233 (2015).

3. Zhang, Q. et al. Md-miR156ab and Md-miR395 target WRKY Transcription factors to influence apple resistance to leaf spot disease. Front. Plant Sci. 8, 526 (2017).

4. Zhang, Q. et al. A single-nucleotide polymorphism in the promoter of a hairpin RNA contributes to Alternaria alternata leaf spot resistance in apple (Malus $\times$ domestica). Plant Cell 30, 1924-1942 (2018).

5. $\mathrm{Hu}, \mathrm{Q}$. et al. Laccase ghlac1 modulates broad-spectrum biotic stress tolerance via manipulating phenylpropanoid pathway and jasmonic acid synthesis. Plant Physiol. 176, 1808-1823 (2018).

6. Boller, T. \& He, S. Y. Innate immunity in plants: an arms race between pattern recognition receptors in plants and effectors in microbial pathogens. Science 324, 742-744 (2009).

7. Dodds, P. N. \& Rathjen, J. P. Plant immunity: towards an integrated view of plant-pathogen interactions. Nat. Rev. Genet. 11, 539-548 (2010).

8. Dangl, J. L., Horvath, D. M. \& Staskawicz, B. J. Pivoting the plant immune system from dissection to deployment. Science 341, 746-751 (2013).

9. Tang, D., Wang, G. \& Zhou, J. M. Receptor kinases in plant-pathogen interactions: more than pattern pecognition. Plant Cell 29, 618-637 (2017).

10. Venkatesh, J. \& Kang, B. C. Current views on temperature-modulated R genemediated plant defense responses and tradeoffs between plant growth and immunity. Curr. Opin. Plant Biol. 50, 9-17 (2019).

11. Ruan, J. et al. Jasmonic acid signaling pathway in plants. Int. J. Mol. Sci. 20, 2479 (2019).

12. hang, Y. \& Li, X. Salicylic acid: biosynthesis, perception, and contributions to plant immunity. Curr. Opin. Plant Biol. 50, 29-36 (2019).

13. Derksen, H., Rampitsch, C. \& Daayf, F. Signaling cross-talk in plant disease resistance. Plant Sci. 207, 79-87 (2013).

14. van Loon, L. C., Rep, M. \& Pieterse, C. M. Significance of inducible defenserelated proteins in infected plants. Annu Rev. Phytopathol. 44, 135-162 (2006).

15. Robert-Seilaniantz, A., Grant, M. \& Jones, J. D. Hormone crosstalk in plant disease and defense: more than just jasmonate-salicylate antagonism. Annu. Rev. Phytopathol. 49, 317-343 (2011).

16. Eulgem, T. \& Somssich, I. E. Networks of WRKY transcription factors in defense signaling. Curr. Opin. Plant Biol. 10, 366-371 (2007).

17. $\mathrm{Xu}$, L. et al. An Arabidopsis mutant cex1 exhibits constant accumulation of jasmonate-regulated AtVSP, Thi2.1 and PDF1.2. FEBS Lett. 494, 161-164 (2001).

18. Brown, R. L., Kazan, K., McGrath, K. C., Maclean, D. J. \& Manners, J. M. A role for the GCC-box in jasmonate-mediated activation of the PDF1.2 gene of Arabidopsis. Plant Physiol. 132, 1020-1032 (2003).

19. Choudhury, F. K., Rivero, R. M., Blumwald, E. \& Mittler, R. Reactive oxygen species, abiotic stress and stress combination. Plant J. 90, 856-867 (2017).

20. Biswas, K. et al. Reactive Oxygen Species and Antioxidant Defence Systems in Plants: Role and Crosstalk Under Biotic Stress in Sustainable Agriculture in the Era of Climate Change. Ch. 12, 265-292 (2020).

21. Seyfferth, C. \& Tsuda, K. Salicylic acid signal transduction: the initiation of biosynthesis, perception and transcriptional reprogramming. Front. Plant Sci. 5, 697 (2014).

22. La Camera, S. et al. Metabolic reprogramming in plant innate immunity: the contributions of phenylpropanoid and oxylipin pathways. Immunol. Rev. 198, 267-284 (2004).

23. Moura, J. C., Bonine, C. A., de Oliveira Fernandes Viana, J., Dornelas, M. C. \& Mazzafera, P. Abiotic and biotic stresses and changes in the lignin content and composition in plants. J. Integr. Plant Biol. 52, 360-376 (2010).

24. Boerjan, W., Ralph, J. \& Baucher, M. Lignin biosynthesis. Annu. Rev. Plant Biol. 54, 519-546 (2003).

25. Eulgem, T., Rushton, P. J., Robatzek, S. \& Somssich, I. E. The WRKY superfamily of plant transcription factors. Trends Plant Sci. 5, 199-206 (2000).

26. Jiang, J. et al. WRKY transcription factors in plant responses to stresses. J. Integr Plant Biol. 59, 86-101 (2017).

27. Choi, $C$. et al. Identification of AtWRKY75 as a transcriptional regulator in the defense response to Pcc through the screening of Arabidopsis activationtagged lines. Plant Biotechnol. Rep. 8, 183-192 (2013). 
28. Choi, C., Park, S., Ahn, I., Bae, S. \& Hwang, D.-J. Generation of Chinese cabbage resistant to bacterial soft rot by heterologous expression of Arabidopsis WRKY75. Plant Biotechnol. Rep. 10, 301-307 (2016).

29. Chen, $X$. et al. Overexpression of AtWRKY28 and AtWRKY75 in Arabidopsis enhances resistance to oxalic acid and Sclerotinia sclerotiorum. Plant Cell Rep. 32, 1589-1599 (2013).

30. Zhang, L., Chen, L. \& Yu, D. Transcription factor WRKY75 interacts with DELLA proteins to affect flowering. Plant Physiol. 176, 790-803 (2018).

31. Guo, P. et al. A tripartite amplification loop involving the transcription factor WRKY75, salicylic acid, and reactive oxygen species accelerates leaf senescence. Plant Cell 29, 2854-2870 (2017).

32. Zhang, Y. et al. PtrWRKY75 overexpression reduces stomatal aperture and improves drought tolerance by salicylic acid-induced reactive oxygen species accumulation in poplar. Environ. Exp. Bot. 176, 104117 (2020).

33. Zhao, K. et al. Functional characterization of poplar WRKY75 in salt and osmotic tolerance. Plant Sci. 289, 110259 (2019).

34. Zhang, F. et al. MdWRKY100 encodes a group I WRKY transcription factor in Malus domestica that positively regulates resistance to Colletotrichum gloeosporioides infection. Plant Sci. 286, 68-77 (2019).

35. Zhu, Y. \& Saltzgiver, M. Transcriptional profiles of Mdwrky33 in apple root in response to infection by Pythium ultimum, abiotic stresses and chemical treatments. Int. J. Phytopathol. 8, 87-100 (2020).

36. Zhao, X. Y. et al. MdWRKY15 improves resistance of apple to Botryosphaeria dothidea via the salicylic acid-mediated pathway by directly binding the MdICS1 promoter. J. Integr. Plant Biol. 62, 527-543 (2020).

37. Zhao, X. Y. et al. MdWRKY46-enhanced apple resistance to Botryosphaeria dothidea by activating the expression of MdPBS3.1 in the salicylic acid signaling pathway. Mol. Plant Microbe Interact. 32, 1391-1401 (2019).

38. Zhao, X. Y. et al. MdHIR4 transcription and translation levels associated with disease in apple are regulated by MdWRKY31. Plant Mol. Biol. 101, 149-162 (2019).

39. Cheng, P. et al. CmBES1 is a regulator of boundary formation in chrysanthemum ray florets. Hortic. Res. 7, 129 (2020).

40. Woudenberg, J. H. et al. Alternaria section alternaria: species, formae speciales or pathotypes? Stud. Mycol. 82, 1-21 (2015).

41. Rushton, P. J., Somssich, I. E., Ringler, P. \& Shen, Q. J. WRKY transcription factors. Trends Plant Sci. 15, 247-258 (2010).

42. Dong, J., Chen, C. \& Chen, Z. Expression profiles of the Arabidopsis WRKY gene superfamily during plant defense response. Plant Mol. Biol. 51, 21-37 (2003).

43. Lui, S. et al. Identification and expression analysis of WRKY transcription factor genes in response to fungal pathogen and hormone treatments in apple (Malus domestica). J. Plant Biol. 60, 215-230 (2017).

44. Encinas-Villarejo, S. et al. Evidence for a positive regulatory role of strawberry (Fragaria $\times$ ananassa) FaWRKY1 and Arabidopsis AtWRKY75 proteins in resistance. J. Exp. Bot. 60, 3043-3065 (2009).

45. Higuera, J. J. et al. The strawberry FaWRKY1 transcription factor negatively regulates resistance to Colletotrichum acutatum in fruit upon infection. Front. Plant Sci. 10, 480 (2019).

46. Lopez-Galiano, M. J. et al. Epigenetic regulation of the expression of WRKY75 transcription factor in response to biotic and abiotic stresses in Solanaceae plants. Plant Cell Rep. 37, 167-176 (2018).

47. Meng, D. et al. Sorbitol modulates resistance to Alternaria alternata by regulating the expression of an NLR resistance gene in apple. Plant Cell 30, 1562-1581 (2018).

48. Loake, G. \& Grant, M. Salicylic acid in plant defence-the players and protagonists. Curr. Opin. Plant Biol. 10, 466-472 (2007)

49. $\mathrm{Wu}, \mathrm{Y}$. et al. The Arabidopsis NPR1 protein is a receptor for the plant defense hormone salicylic acid. Cell Rep. 1, 639-647 (2012).

50. Chico, J. M., Chini, A., Fonseca, S. \& Solano, R. JAZ repressors set the rhythm in jasmonate signaling. Curr. Opin. Plant Biol. 11, 486-494 (2008).

51. Wang, F. et al. Overexpression of Chitinase gene enhances resistance to Colletotrichum gloeosporioides and Alternaria alternata in apple (Malus $\times$ domestica). Sci. Hortic. 277, 109779 (2021).

52. Liu, Y., Liu, Q., Tang, Y. \& Ding, W. NtPR1a regulates resistance to Ralstonia solanacearum in Nicotiana tabacum via activating the defense-related genes. Biochem. Biophys. Res. Commun. 508, 940-945 (2019).

53. Li, J., Brader, G., Kariola, T. \& Palva, E. T. WRKY70 modulates the selection of signaling pathways in plant defense. Plant J. 46, 477-491 (2006).
54. Mao, P., Duan, M., Wei, C. \& Li, Y. WRKY62 transcription factor acts downstream of cytosolic NPR1 and negatively regulates jasmonate-responsive gene expression. Plant Cell Physiol. 48, 833-842 (2007).

55. Singh, A., Sarma, B. K., Upadhyay, R. S. \& Singh, H. B. Compatible rhizosphere microbes mediated alleviation of biotic stress in chickpea through enhanced antioxidant and phenylpropanoid activities. Microbiol. Res. 168, 33-40 (2013).

56. You, X., Fang, H., Wang, R., Wang, G.-L. \& Ning, Y. Phenylalanine ammonia lyases mediate broad-spectrum resistance to pathogens and insect pests in plants. Sci. Bull. 65, 1425-1427 (2020).

57. Devaiah, B. N., Karthikeyan, A. S. \& Raghothama, K. G. WRKY75 transcription factor is a modulator of phosphate acquisition and root development in Arabidopsis. Plant Physiol. 143, 1789-1801 (2007)

58. Rishmawi, L. et al. Non-cell-autonomous regulation of root hair patterning genes by WRKY75 in Arabidopsis. Plant Physiol. 165, 186-195 (2014).

59. Zheng, $X$. et al. MdWRKY9 overexpression confers intensive dwarfing in the M26 rootstock of apple by directly inhibiting brassinosteroid synthetase MdDWF4 expression. N. Phytol. 217, 1086-1098 (2018).

60. Zhang, S. et al. Genomic variants of genes associated with three horticultural traits in apple revealed by genome re-sequencing. Hortic. Res. 1, 14045 (2014).

61. Livak, K. J. \& Schmittgen, T. D. Analysis of relative gene expression data using real-time quantitative PCR and the $2^{-\Delta \Delta C t}$ Method. Methods 25, 402-408 (2001).

62. Zhou, F. et al. D14-SCF ${ }^{\mathrm{D} 3}$-dependent degradation of D53 regulates strigolactone signalling. Nature 504, 406-410 (2013).

63. Horsch, R. B. et al. A simple and general method for transferring genes into plants. Science 227, 1229-1231 (1985).

64. Dai, $\mathrm{H}$. et al. Development of a seedling clone with high regeneration capacity and susceptibility to Agrobacterium in apple. Sci. Hortic. 164, 202-208 (2013).

65. Zhou, K. et al. MdUGT88F1-mediated phloridzin biosynthesis regulates apple development and valsa canker resistance. Plant Physiol. 180, 2290-2305 (2019).

66. Geng, D. et al. Regulation of phenylpropanoid biosynthesis by MdMYB88 and MdMYB124 contributes to pathogen and drought resistance in apple. Hortic. Res. 7, 102 (2020)

67. Sun, C. et al. The R2R3-type MYB transcription factor MdMYB90-like is responsible for the enhanced skin color of an apple bud sport mutant. Hortic. Res. 8, 156 (2021).

68. LV, Y., Zhang, M., Wu, T., Wu, T. \& Zhong, Y. The infiltration efficiency of Agrobacterium-mediated transient transformation in four apple cultivars. Sci. Hortic. 256, 108597 (2019).

69. Zhou, H. et al. CRISPR/Cas9-mediated mutagenesis of MdCNGC2 in apple callus and VIGS-mediated silencing of MdCNGC2 in fruits improve resistance to Botryosphaeria dothidea. Front. Plant Sci. 11, 575477 (2020).

70. Hellens, R. P. et al. Transient expression vectors for functional genomics, quantification of promoter activity and RNA silencing in plants. Plant Methods 1, 13 (2005).

71. Sparkes, I. A., Runions, J., Kearns, A. \& Hawes, C. Rapid, transient expression of fluorescent fusion proteins in tobacco plants and generation of stably transformed plants. Nat. Protoc. 1, 2019-2025 (2006).

72. Xu, J., Lee, Y. J. \& Liu, B. Establishment of a mitotic model system by transient expression of the D-type cyclin in differentiated leaf cells of tobacco (Nicotiana benthamiana). N. Phytol. 226, 1213-1220 (2020).

73. Liu, Y. et al. A WRKY transcription factor PbrWRKY53 from Pyrus betulaefolia is involved in drought tolerance and AsA accumulation. Plant Biotechnol. J. 17, 1770-1787 (2019)

74. Kumar, D., Yusuf, M. A., Singh, P., Sardar, M. \& Sarin, N. B. Histochemical detection of superoxide and $\mathrm{H}_{2} \mathrm{O}_{2}$ accumulation in Brassica juncea seedlings. Bio-Protoc. 4, e1108 (2014).

75. Sun, X. et al. Improvement of drought tolerance by overexpressing MdATG18a is mediated by modified antioxidant system and activated autophagy in transgenic apple. Plant Biotechnol. J. 16, 545-557 (2018).

76. Nakashima, J., Chen, F., Jackson, L., Shadle, G. \& Dixon, R. A. Multi-site genetic modification of monolignol biosynthesis in alfalfa (Medicago sativa): effects on lignin composition in specific cell types. N. Phytol. 179, 738-750 (2008).

77. Zhang, F. J., Jin, Y. J., Xu, X. Y., Lu, R. C. \& Chen, H. J. Study on the extraction, purification and quantification of jasmonic acid, abscisic acid and indole-3acetic acid in plants. Phytochem. Anal. 19, 560-567 (2008).

78. Pan, X., Welti, R. \& Wang, X. Quantitative analysis of major plant hormones in crude plant extracts by high-performance liquid chromatography-mass spectrometry. Nat. Protoc. 5, 986-992 (2010). 